\title{
General model for the kinetics of solute diffusion at solid-solid interfaces
}

\author{
Fernando D. León-Cázares $\circledast^{1, *}$ and Enrique I. Galindo-Nava $\odot^{2,1, \dagger}$ \\ ${ }^{1}$ Department of Materials Science and Metallurgy, University of Cambridge, 27 Charles Babbage Rd, Cambridge, CB3 OFS, United Kingdom \\ ${ }^{2}$ Department of Mechanical Engineering, University College London, Torrington Place, London, WC1E 7JE, United Kingdom
}

(Received 5 July 2021; revised 31 October 2021; accepted 2 December 2021; published 16 December 2021)

\begin{abstract}
Solute diffusion through solid-solid interfaces is paramount to many physical processes. From a modeling point of view, the discontinuities in the energy landscape at a sharp interface represent difficulties in predicting solute diffusion that, to date, have not been solved in a consistent manner across length scales. Using an explicit finite volume method, this work is the first to derive numerical solutions to the diffusion equations at a continuum level while including discrete variations in the energy landscape at a bicrystal interface. An atomic jump equation consistent with atomistic descriptions is derived and scaled up into a compendium of model interfaces: monolayer energy barriers, monolayer interfacial traps, multilayered traps, and heterogeneous interfaces. These can track solute segregation behavior and long-range diffusion effects. We perform simulations with data for hydrogen diffusion in structural metals, of relevance to the assessment of the hydrogen embrittlement phenomenon, and point defects in electronic devices. The approach developed represents an advancement in the mathematical treatment of solute diffusion through solid-solid interfaces and an important bridge between the atomistic and macroscopic modeling of diffusion, with potential applications in a variety of fields in materials science and physics.
\end{abstract}

DOI: 10.1103/PhysRevMaterials.5.123802

\section{INTRODUCTION}

There are different solid-solid interfaces in crystalline materials, such as grain and twin boundaries, stacking faults or interfaces between any two arbitrary phases, and they influence the overall mass diffusion kinetics. For instance, grain boundaries can affect the rate at which thermochemical treatments, such as nitriding [1] or boriding [2] occur, or act as channels of fast diffusion for other interstitial impurities (e.g., [3-5]). In the context of ionic diffusion, interfaces can also act as energy barriers for atomic migrations in solid-state batteries [6,7] and proton conducting fuel cells [8]. In addition, often certain elements preferentially segregate to or away from these interfaces, which can have an impact in materials properties. For example, accumulation of hydrogen at these regions can locally lower the cohesive strength, promoting the formation of intergranular cracks $[9,10]$ and interface decohesion [11]. Similarly, elemental segregation to stacking faults and microtwins in nickel-based superalloys facilitates creep deformation by lowering the stress required to propagate these faults $[12,13]$. A key factor to consider when studying the kinetics of solute diffusion of a system, is that often the atomic behaviours near interfaces are very different to those in the bulk. Thus there is a need for models that can predict the effects of solid-solid interfaces on both long-range diffusion effects and localized segregation.

The interfaces considered here are those in which there is a clear separation between the two neighboring crystals.

\footnotetext{
*fd122@cam.ac.uk

†e.galindo-nava@ucl.ac.uk
}

These are assumed to remain immobile within the time frames considered, either because the solute has a negligible effect on the local phase stability or because the transformation kinetics are considerably slower than diffusion of the solute. This is in contrast with the behavior of diffuse interfaces that develop via interdiffusion of atoms across two materials.

Diffusion of a component occurs in the direction of its decreasing chemical potential, as first shown experimentally over seventy years ago in the seminal work of Darken [14]. Since then, multiple approaches have been suggested to model diffusion in multiphase systems. Works by Cahn [15] and Hillert [16] considered the effects of concentration inhomogeneities by first determining the steady state solution which minimized the free energy of the system. The kinetics were then built such that, in the absence of inhomogeneities, the models became consistent with the macroscopic transport equations of Darken [17]. Atomic mobilities have since been linked to comprehensive CALPHAD thermodynamic databases to model diffusion in multicomponent solutions $[18,19]$. These approaches excel at capturing the concentration dependencies of the driving forces over arbitrarily large length scales but ignore the local variations in thermodynamic and kinetic parameters intrinsic to interfaces.

At the atomic scale, diffusion is dictated by the energy landscape of the solute species embedded in a crystal. The thermodynamic principles of segregation at surfaces and interfaces were first derived by McLean [20]. While variations in the energy landscape often occur over a number of atomic layers in the vicinity of interfaces, as shown in several first-principle studies [21-23], a monolayer approximation was adopted for simplicity. Models such as that by Lea and Seah [24] attempted to capture the associated kinetics at a 
continuum level, which ignored any existing heterogeneity in the energy barriers. To address this, Hofmann and Erlewein [25] modeled diffusion at the atomic scale based on reaction rate theory, using systems of equations that consider the atomic jumping ratios layer-by-layer. Similar models coupled this approach with the mobility based equations of Darken for binary alloys [26-28] and multicomponent systems [29], and stochastic derivations validated this approximation under the assumption of a local average chemical potential [30]. However, all these models lack scalability for long-range diffusion effects and cannot be generalized for arbitrary bicrystal interfaces maintaining a thermodynamic equilibrium. The former has been improved upon by coupling the atomic jump equations at grain boundaries with a finite element method in the bulk [31], but only for bicrystal configurations of a single material.

The modeling of hydrogen diffusion in structural alloys, examples of which are developed in Sec. III of the current work, often suffers from such drawbacks. This field is technologically relevant due to the need to understand and mitigate the phenomenon of $\mathrm{H}$ embrittlement, a process by which the presence of $\mathrm{H}$ in the lattice results in a loss of ductility in the material [32]. Grain boundaries and other bicrystal interfaces influence its kinetics of diffusion, with important implications for alloy design and in-service life of components in $\mathrm{H}$-rich environments [33,34]. The thermodynamics of $\mathrm{H}$ at or near such interfaces are often obtained via density functional theory (DFT) (e.g., [21-23,35]), but their influence on the transient behaviours of segregation and long-range diffusion effects remains inaccessible at larger length scales for arbitrary energy landscapes.

The current work introduces a one-dimensional model of diffusion and segregation at an immobile solid-solid interface that is thermodynamically consistent, acknowledges the local variations in the energy landscape and is fully scalable across length scales. The monolayer approach is developed for the cases of energy barriers and interfacial traps, and later generalized for multilayered and heterogeneous interfaces. Finally, we develop examples with diffusion data of $\mathrm{H}$ in structural metals and point defects in electronic devices, as well as parametric analyses. The numerical methods developed capture interfacial phenomena at the atomic scale for a variety of scenarios in scalable computationally inexpensive algorithms. This fills an important previously missing link between atomistic and macroscopic diffusion modeling.

\section{MODELING}

\section{A. Thermodynamic equilibrium}

Equations for interfacial segregation under equilibrium are based on the seminal work of McLean [20], later expanded upon by du Plessis and van Wyk [36,37] and Lejček [38], amongst others. These were derived for a thin grain boundary and semi-infinite bulk grains [36], although their application to any two different regions is equally valid, as shown next.

Consider a system in thermodynamic equilibrium of two neighboring regions, $\mathrm{A}$ and $\mathrm{B}$, with $m$ substitutional or $m-1$ interstitial diffusing species. Note that all following equations apply for both scenarios by substituting any term related to the host element with a corresponding one for an empty interstitial site. The Gibbs free energy of such system is

$$
G=\sum_{j=1}^{m} \mu_{A}^{(j)} n_{A}^{(j)}+\sum_{j=1}^{m} \mu_{B}^{(j)} n_{B}^{(j)}
$$

where $n_{i}^{(j)}$ is the number of moles of species $j$ in region $i$ and $\mu_{i}^{(j)}$ their chemical potential. A minimum in the Gibbs free energy characterizes the equilibrium point, i.e.,

$$
\frac{\partial G}{\partial n_{i}^{(j)}}=0
$$

where all the terms containing $\partial \mu_{i}^{(j)}$ in the derivative cancel out due to the Gibbs-Duhem equation. A couple of constraints related to mass conservation reduce the number of equations in relation (2) [36]. The total number of moles in each region remains constant, so that of the host element (or the empty interstitial site) $n_{i}^{(m)}$ can be expressed in terms of all others. Its time evolution results in $\partial n_{i}^{(m)} / \partial n_{i}^{(j)}=-1$ for any $i \neq m$. Similarly, all fluxes occur between regions $\mathrm{A}$ and $\mathrm{B}$, which can be expressed as $\partial n_{B}^{(j)} / \partial n_{A}^{(j)}=-1$. Differentiating Eq. (1), accounting for these constraints, and substituting into (2) results in $m-1$ equations

$$
\left(\mu_{A}^{(j)}-\mu_{A}^{(m)}\right)-\left(\mu_{B}^{(j)}-\mu_{B}^{(m)}\right)=0
$$

for $j=1,2, \ldots, m-1$. Equation (3) is analogous to that in Ref. [36] but derived for any two regions of arbitrary size and arranged by region rather than by species.

The multisolute Langmuir-McLean segregation isotherms can be obtained by substituting into Eq. (3) the expression for the chemical potential

$$
\mu_{i}^{(j)}=\mu_{i 0}^{(j)}+R T \ln a_{i}^{(j)}
$$

where $\mu_{i 0}^{(j)}$ is the standard chemical potential, $a_{i}^{(j)}=\gamma_{i}^{(j)} X_{i}^{(j)}$ the thermodynamic activity, $\gamma_{i}^{(j)}$ the activity coefficient, $X_{i}^{(j)}$ the atomic fraction, $R$ the gas constant and $T$ the absolute temperature of the system, leading to

$$
\begin{aligned}
& \frac{\gamma_{A}^{(j)}}{\gamma_{A}^{(m)}} \frac{X_{A}^{(j)}}{X_{A}^{(m)}} \\
& \quad=\frac{\gamma_{B}^{(j)}}{\gamma_{B}^{(m)}} \frac{X_{B}^{(j)}}{X_{B}^{(m)}} \exp \left(-\frac{\left(\mu_{A 0}^{(j)}-\mu_{A 0}^{(m)}\right)-\left(\mu_{B 0}^{(j)}-\mu_{B 0}^{(m)}\right)}{R T}\right) .
\end{aligned}
$$

Rearranging terms and considering that $\sum_{j=1}^{m} X_{i}^{(j)}=1$, this equation can be rewritten as

$$
\frac{X_{A}^{(j)}}{1-\sum_{k=1}^{m-1} X_{A}^{(k)}}=\frac{X_{B}^{(j)}}{1-\sum_{k=1}^{m-1} X_{B}^{(k)}} \exp \left(-\frac{\Delta G_{0}^{(j)}+\Delta G_{E}^{(j)}}{R T}\right),
$$

in agreement with previous studies [38,39]. The two terms inside the exponential function in (6) are the molar Gibbs free 
energy of segregation for an ideal solution

$$
\Delta G_{0}^{(j)}=\left(\mu_{A 0}^{(j)}-\mu_{A 0}^{(m)}\right)-\left(\mu_{B 0}^{(j)}-\mu_{B 0}^{(j m)}\right)
$$

and an excess term

$$
\Delta G_{E}^{(j)}=R T \ln \left(\frac{\gamma_{A}^{(j)} \gamma_{B}^{(m)}}{\gamma_{A}^{(m)} \gamma_{B}^{(j)}}\right)
$$

that accounts for the solute-solute interactions in a real solution. See Ref. [40,41] for a more detailed explanation of these parameters. A simplified form of Eq. (6) for a binary ideal and dilute system is then

$$
X_{A}^{(1)} \approx X_{B}^{(1)} \exp \left(-\frac{\Delta G_{0}^{(1)}}{R T}\right),
$$

which will be useful for the derivation of the diffusion kinetics.

An extension of the above derivation to more than two neighboring regions in space follows the same relations from Eq. (3) for each diffusing species, regardless of their relative sizes. As such, this applies to specific sites (point traps), interfaces and large volumes. As an ergodic system, the average occupancy of a site is a function of the energy landscape [42].

\section{B. Kinetics of diffusion}

This section is restricted for simplicity to the case of onedimensional diffusion with one solute (species 1 and $m$, with the superscript 1 avoided hereafter whenever possible). The evolution of the concentration $c(x, t)$ at any given point in space $x$ and time $t$ is given by Fick's second law of diffusion

$$
\frac{\partial}{\partial t} c(x, t)=-\frac{1}{\psi} \frac{\partial}{\partial x} \psi J(x, t),
$$

where $\psi=1$ for cartesian coordinates, $\psi=x$ for cylindrical coordinates and $\psi=x^{2}$ for spherical coordinates, $x$ refers in the latter two cases to the radial direction [43], and $J(x, t)$ is the solute flux. Curved solid-solid interfaces are rarely homogeneous. Nonetheless, the derivations are also performed for these coordinate systems as they may be useful simplifications for the analysis of some scenarios, e.g., solute diffusion in and out of fibres or spherical precipitates assuming that the interface is homogeneous. The discontinuities at an interface demand the use of chemical potential-driven equations, i.e..

$$
J(x, t)=-M(x) c(x, t) \frac{d}{d x} \mu(x, t),
$$

where $M=D / R T$ is the mobility, $D=D_{0} \exp (-Q / R T)$ the coefficient of diffusion, $D_{0}$ its maximum value possible, $Q$ the energy barrier between neighboring atomic sites, and $\mu=\mu^{(1)}-\mu^{(m)}$. As expected from Eq. (3), a gradient in the chemical potential that considers both species is the driving force for diffusion, and combined with Eq. (4) and relation $X^{(j)}=c^{(j)} / S$ it can be expressed as

$$
\mu(x, t)=E(x)+R T \ln \frac{\gamma^{(1)}(x) c(x, t)}{\gamma^{(m)}(x)(S(x)-c(x, t))},
$$

where $S$ is the number density of available sites. The energy $E$ in Eq. (12) corresponds to the difference in standard chemical potentials that appears in Eq. (7). As pointed out by Lejček et al. [44], density functional theory and atomistic simulations often calculate this energy difference from two computational cells, with and without the solute, instead of the actual Gibbs free energies $G_{0}^{(j)}$. However, the difference between these parameters is negligible under normal pressures; thus

$$
E_{i}^{(j)} \approx G_{i 0}^{(j)}=\mu_{i 0}^{(j)}-\mu_{i 0}^{(m)} .
$$

Assuming an ideal dilute solution, i.e., $\gamma^{(1)}=\gamma^{(m)}=1$ and $S \gg c$, and combining Eqs. (11) and (12) yields

$$
J(x, t)=-D(x)\left(\frac{\partial}{\partial x} c(x, t)+\frac{c(x, t)}{R T} \frac{\partial E(x)}{\partial x}-\frac{c(x, t)}{S(x)} \frac{\partial S(x)}{\partial x}\right) .
$$

It is the dilute solution assumption which later reduces all the analytical expressions found back to the original Fick's laws of diffusion. The difficulty in obtaining the flux from Eq. (14) lies in the discontinuity of the parameters $D, E$, and $S$ at a sharp interface. This renders analytical solutions to Eq. (10) infeasible. Numerical solutions for the evolution of the concentration profiles are sought instead.

Explicit finite volume methods (FVM) on equispaced grids are implemented here to approximate the solution to this partial differential equation (PDE) in cartesian, cylindrical or spherical coordinates. This integral formulation has the advantage of conserving the solute and accounting for the discontinuities $[45,46]$, while keeping the computational time considerably small. The space domain is subdivided into control volumes of width $\Delta x$ denominated cells, with centres situated at coordinates $x_{i}$, boundaries at $x_{i \pm \frac{1}{2}}$, boundary areas $A_{i \pm \frac{1}{2}}$ and volumes $V_{i}(i=1,2, \ldots, n)$. A weak formulation of Eq. (10) becomes

$$
\int_{V_{i}} \frac{\partial c}{\partial t} d V=-\int_{V_{i}} \frac{1}{\psi} \frac{\partial}{\partial x} \psi J d V
$$

for each cell. Using a forward difference in time intervals of $\Delta t$ duration and employing Gauss's divergence theorem on the right-hand side of Eq. (15) results in

$$
\frac{c_{i}^{k+1}-c_{i}^{k}}{\Delta t}=\frac{J_{i-\frac{1}{2}}^{k} A_{i-\frac{1}{2}}-J_{i+\frac{1}{2}}^{k} A_{i+\frac{1}{2}}}{V_{i}},
$$

where $c_{i}^{k}$ is the average concentration of cell $i$ at time step $k$. The fluxes at the individual boundaries $J_{i \pm \frac{1}{2}}^{k}$ can in return be reconstructed from the cell averages. When both cells next to a boundary and the boundary itself belong to a homogeneous material, the flux is

$$
J_{i-\frac{1}{2}}^{k}=-D \frac{c_{i}^{k}-c_{i-1}^{k}}{\Delta x}
$$




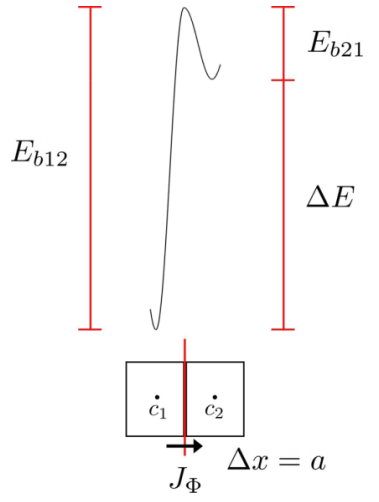

FIG. 1. Energy landscape (top) and cell discretization (bottom) of an atomic jump configuration.

which combined with Eq. (16) results in a numerical method identical to an explicit finite differences scheme. As such, the criterion for convergence and numerical stability $D \Delta t / \Delta x^{2} \leqslant 1 / 2$ also applies for this FVM approach [43]. Note that Eq. (16) applies for the three coordinate systems and accounts for the parameter $\psi$ via the cell areas and volumes. All that is left to complete the iterative solution is determining the flux and solute redistribution at the cells in the vicinity of the interface.

\section{Atomic jumps}

It is necessary to start the analysis at the smallest length scale relevant to the study of diffusion in crystals, i.e., an atomic jump. Unlike the models of Hofmann and Erlewein [25], Martin [30], and Ilin et al. [31], all based in layer-bylayer atomic configurations and jumping rates, the approach here can be understood as a discretized form of the continuous Darken differential equations [17]. The prefactor of the coefficient of diffusion is the link between the at- tempt frequencies and the macroscopic diffusivities in these approaches.

Consider two neighboring cells $(i=1,2)$ with thickness of a single atomic layer $\Delta x=a$ and each with its own concentration, thermodynamic $\left(E_{i}, S_{i}\right)$ and kinetic $\left(D_{0 i}\right)$ parameters, as shown in Fig. 1. These may correspond to any arbitrary sites, be it in the bulk or at an interface. Diffusion between these cells is affected by the difference in energy states $\Delta E=$ $E_{2}-E_{1}$ and the corresponding asymmetrical energy barrier $E_{b 12}=\Delta E+E_{b 21}$. The saddle point $\Phi$ is assumed to be situated exactly in the middle between both sites, which is required to address the ill-defined diffusivity prefactors between two very different lattice (or interstitial) sites in a continuous framework. This assumption has no effect on the diffusion in the bulk.

Within the context of a continuous function that is discretized in a FVM model, the variation in chemical potential across the saddle point will not be linear, as it is often wrongly assumed (e.g., Ref. [47]), unless both sites are identical in nature. Instead, a condition of equal flux on both sides of $J_{\Phi}=J_{\Phi^{-}}=J_{\Phi^{+}}$is necessary [48,49], where

$$
\begin{aligned}
& J_{\Phi^{-}}=-D_{01} \exp \left(-\frac{E_{b 12}}{R T}\right) \frac{c_{\Phi^{-}}-c_{1}}{a / 2}, \\
& J_{\Phi^{+}}=-D_{02} \exp \left(-\frac{E_{b 21}}{R T}\right) \frac{c_{2}-c_{\Phi^{+}}}{a / 2},
\end{aligned}
$$

and the concentrations $c_{\Phi^{ \pm}}$are those on each side of the interface, given by Eq. (4) as

$$
c_{\Phi}=S \exp \left(\frac{\mu_{\Phi}-E}{R T}\right)
$$

with their respective $E$ and $S$ values. Solving Eqs. (18a) and (18b) yields the chemical potential at the saddle point

$$
\mu_{\Phi}=E_{1}+R T \ln \left(\frac{c_{1} D_{01} \exp \left(-\frac{E_{b 12}}{R T}\right)+c_{2} D_{02} \exp \left(-\frac{E_{b 21}}{R T}\right)}{S_{1} D_{01} \exp \left(-\frac{E_{b 12}}{R T}\right)+S_{2} D_{02} \exp \left(-\frac{E_{b 21}+\Delta E}{R T}\right)}\right)
$$

and the flux through it

$$
J_{\Phi}=-\hat{D}_{b} \frac{\frac{c_{2}}{S_{2}}-\frac{c_{1}}{S_{1}} \exp \left(-\frac{\Delta E}{R T}\right)}{a},
$$

with

$$
\hat{D}_{b}=\frac{2 S_{1} S_{2} D_{01} D_{02}}{S_{1} D_{01}+S_{2} D_{02}} \exp \left(-\frac{E_{b 21}}{R T}\right) .
$$

The flux in Eq. (21) consists of an equivalent coefficient of diffusion $\hat{D}_{b}$ for the atomic jumps and a dimensionless form of the driving force. Note that these concentrations are normalized with respect to their solubilities and the energies referenced to cell 2 via the exponential factor. Hereafter, all dimensionless driving forces will be referenced to the rightmost energy level for consistency, unless otherwise specified. Their associated equivalent coefficients of diffusion, with units $\mathrm{mol} \mathrm{m}^{-1} \mathrm{~s}^{-1}$, will be denoted by the caret symbol(^)${ }^{\wedge}$.

\section{Monolayer energy barrier}

Consider the simplest bicrystal interface between two dissimilar materials, shown in Fig. 2(a). A common approach consists in employing the conditions of equal flux and chemical potential at the interface used in the previous section, but over arbitrarily large cells of length $\Delta x$. Having the interface situated at point $x_{\Phi}$ between two cells, this results in two equations $J_{\Phi^{-}}=-D_{1} \frac{c_{\Phi^{-}}-c_{1}}{\Delta x / 2}$ and $J_{\Phi^{+}}=-D_{2} \frac{c_{2}-c_{\Phi^{+}}}{\Delta x / 2}$, which yield an overall flux

$$
J_{\Phi}=-\hat{D}_{b i} \frac{\frac{c_{2}}{S_{2}}-\frac{c_{1}}{S_{1}} \exp \left(-\frac{\Delta E}{R T}\right)}{\Delta x}
$$

with an effective coefficient of diffusion for the bicrystal interface of

$$
\hat{D}_{b i}=\left(\frac{1}{2 S_{1} D_{1}} \exp \left(-\frac{\Delta E}{R T}\right)+\frac{1}{2 S_{2} D_{2}}\right)^{-1} .
$$


(a)

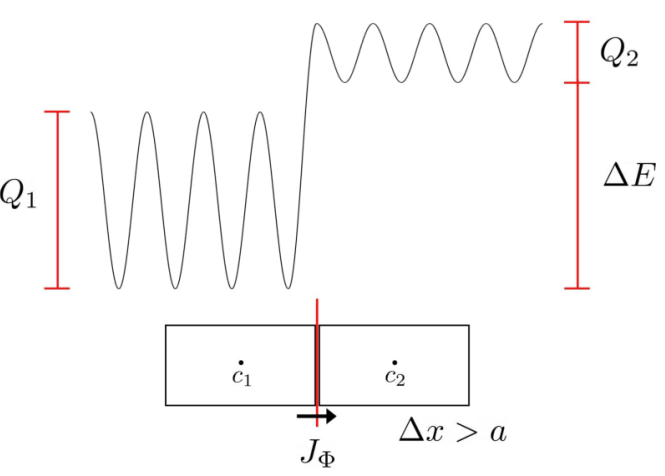

(b)

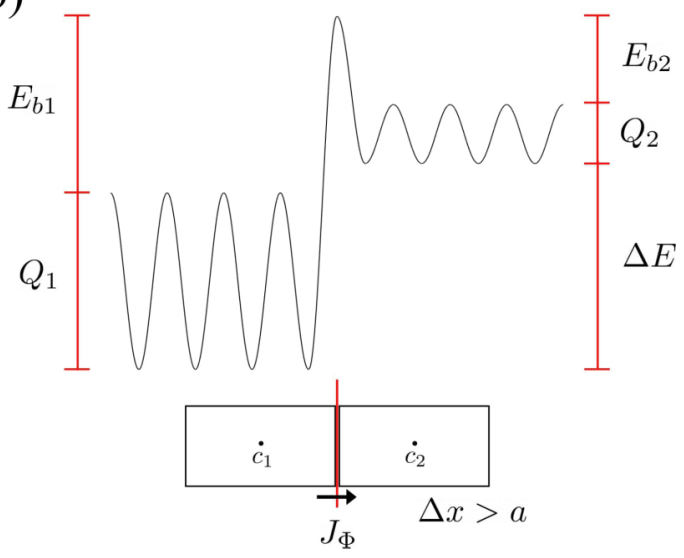

FIG. 2. Energy landscapes (top) and cell discretizations (bottom) of a bicrystal interface (a) without an additional energy barrier and (b) with an arbitrary energy barrier.

However, this result does not consider the atomic jump at the interface. More importantly, it does not allow for the consideration of any localized variation in the energy landscape near the interface.

Thus we tackle the more general geometry in Fig. 2(b), where the interface $\Phi$ separating two crystals 1 and 2 has an arbitrarily large energy barrier. By aligning the interface and the cell boundary we ensure that there are no large variations in the properties within the cells, so that the weak formulation in Eq. (15) holds. To the knowledge of the authors, there is currently no model that accounts for such variation in the energy landscape while capturing other long-range diffusion effects in the neighboring crystals. Note that the case of $E_{b 2}=0$ is equivalent to that in Fig. 2(a), so all cases can be treated in the same way.

Similar to the analysis in Sec. IIB1, a condition of equal flux must be attained in the vicinity of the interface. The length fractions of the three relevant regions are $\phi_{1}=\frac{1}{2}-\frac{a}{2 \Delta x}$ for crystal $1, \phi_{b}=\frac{a}{\Delta x}$ for the length of an atomic jump through the interface and $\phi_{2}=\frac{1}{2}-\frac{a}{2 \Delta x}$ for crystal 2. The corresponding fluxes are

$$
\begin{aligned}
& J_{1}=-D_{1} \frac{c_{\Phi 1}-c_{I-1}}{\phi_{1} \Delta x}, \\
& J_{b}=-\hat{D}_{b} \frac{\frac{c_{\Phi 2}}{S_{2}}-\frac{c_{\Phi 1}}{S_{1}} \exp \left(-\frac{\Delta E}{R T}\right)}{\phi_{b} \Delta x}, \\
& J_{2}=-D_{2} \frac{c_{I}-c_{\Phi 2}}{\phi_{2} \Delta x},
\end{aligned}
$$

where $c_{\Phi 1}$ and $c_{\Phi 2}$ are the concentrations at the first sites to the left and right of the interface, respectively, and $\hat{D}_{b}$ is given by Eq. (22) with $E_{b 12}=Q_{1}+E_{b 1}$ and $E_{b 21}=Q_{2}+E_{b 2}$. Equating these fluxes $J_{1}=J_{b}=J_{2}$, solving for the unknown concentrations and substituting back into Eq. (25) results in a flux

$$
J_{\Phi}=-\hat{D}_{e b} \frac{\frac{c_{2}}{S_{2}}-\frac{c_{1}}{S_{1}} \exp \left(-\frac{\Delta E}{R T}\right)}{\Delta x}
$$

where

$$
\hat{D}_{e b}=\left(\frac{\phi_{1}}{S_{1} D_{1}} \exp \left(-\frac{\Delta E}{R T}\right)+\frac{\phi_{b}}{\hat{D}_{b}}+\frac{\phi_{2}}{S_{2} D_{2}}\right)^{-1}
$$

is the effective coefficient of diffusion for an arbitrary energy barrier.

Compared to Eq. (24), the coefficient of diffusion in Eq. (27) has an additional term that accounts for the energy barrier at the interface. This does not disappear even as $E_{b 2}$ tends to zero, as a barrier $E_{b 1}>0$ remains in place. Further discussions on the validity of both approaches are given in Sec. III A.

\section{Monolayer interfacial trap}

An additional complexity arises when considering a monolayer trap of thickness $a$, as two discontinuities are situated in close proximity to each other, i.e., the energy barriers on both sites of the interface. Tackling this with an intermediate cell of size equal to the trap width would converge to the solution, but at the expense of scalability due to the dependence of the convergence parameter on $\Delta x$. Instead, the approach pursued subdivides the interface cell to make sure that the weak formulation in Eq. (15) holds.

Consider a constant mesh spacing with an interfacial trap of energy $E_{t}=E_{1}-\Delta E_{1 t}$ (or $E_{t}=E_{2}-\Delta E_{2 t}$ ), diffusivity prefactor $D_{0 t}$ and site density $S_{t}$, placed at $x=x_{I}$ at the center of cell $I$, with the geometry and energy landscape shown in Fig. 3(a). Arbitrary energy barriers on each side of the trap, $E_{b 1 t}$ and $E_{b 2 t}$, are added for generality. To determine the flux through its left boundary $J_{I-\frac{1}{2}}$, the length fractions of the two regions to consider are $\phi_{1}=1-\frac{a}{\Delta x}$ for crystal 1 and $\phi_{b 1}=\frac{a}{\Delta x}$ for the length of an atomic jump through the left interface of the trap. Equating the fluxes through both regions yields

$$
c_{\Phi 1}=\frac{S_{I-1}\left(c_{t} \hat{D}_{b 1} \phi_{1} \exp \left(-\frac{\Delta E_{1 t}}{R T}\right)+c_{I-1} S_{t} D_{I-1} \phi_{b 1}\right)}{S_{t} \hat{D}_{b 1} \phi_{1}+S_{I-1} S_{t} D_{I-1} \phi_{b 1}}
$$

and

$$
J_{I-\frac{1}{2}}=-\frac{S_{I-1} D_{I-1} \hat{D}_{b 1}}{\hat{D}_{b 1} \phi_{1}+S_{I-1} D_{I-1} \phi_{b 1}} \frac{\frac{c_{t}}{S_{t}} \exp \left(-\frac{\Delta E_{1 t}}{R T}\right)-\frac{c_{I-1}}{S_{I-1}}}{\Delta x},
$$

where $c_{t}$ is the concentration at the trap site (the notation $c_{I}$ is avoided as it could be confused for a concentration in the entirety of cell $I$ ). A similar analysis for the right boundary of cell $I$, where the regions $\phi_{2}=\phi_{1}$ and $\phi_{b 2}=\phi_{b 1}$, leads to

$$
c_{\Phi 2}=\frac{S_{I+1}\left(c_{I+1} S_{t} D_{I+1} \phi_{b 2}+c_{t} \hat{D}_{b 2} \phi_{2} \exp \left(-\frac{\Delta E_{2 t}}{R T}\right)\right)}{S_{I+1} S_{t} D_{I+1} \phi_{b 2}+S_{t} \hat{D}_{b 2} \phi_{2}}
$$



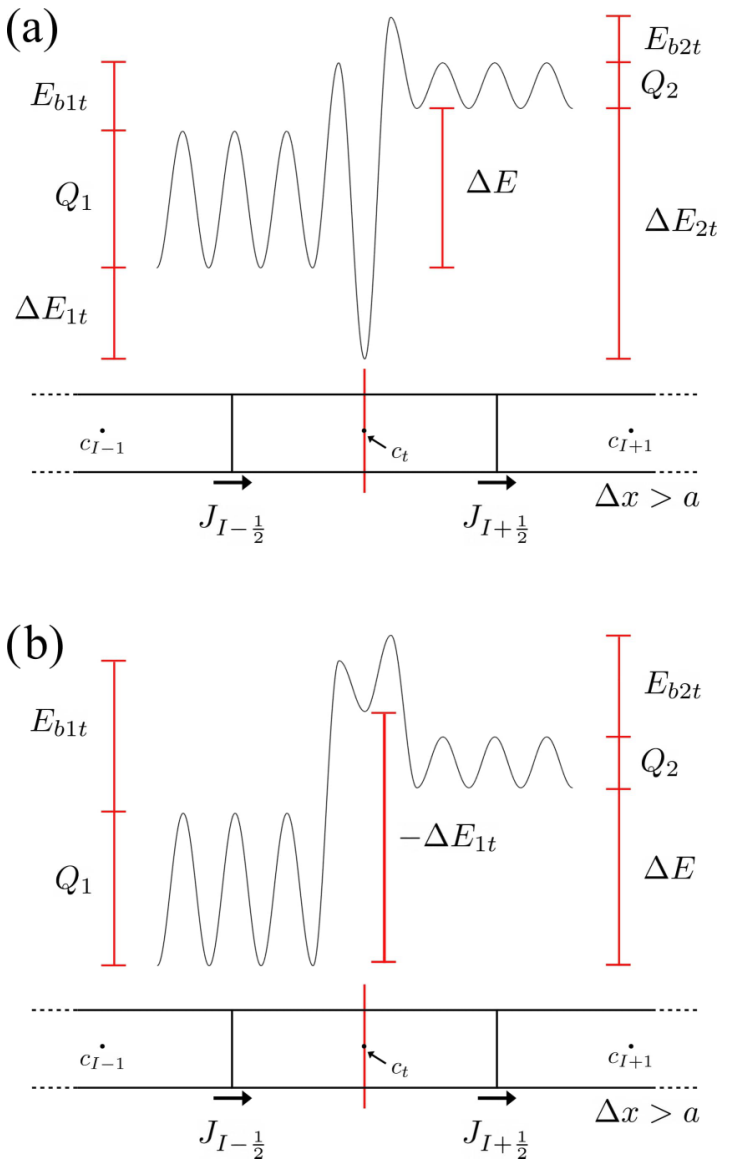

FIG. 3. Energy landscapes (top) and cell discretizations (bottom) of (a) a bicrystal interfacial trap with positive segregation and (b) a bicrystal interfacial energy barrier with sites that undergo negative segregation.

and

$$
J_{I+\frac{1}{2}}=-\frac{S_{I+1} D_{I+1} \hat{D}_{b 2}}{S_{I+1} D_{I+1} \phi_{b 2}+\hat{D}_{b 2} \phi_{2}} \frac{\frac{c_{I+1}}{S_{I+1}}-\frac{c_{t}}{S_{t}} \exp \left(-\frac{\Delta E_{2 t}}{R T}\right)}{\Delta x} .
$$

The coefficients of diffusion $\hat{D}_{b i}(i=1,2)$ are those obtained from Eq. (22) for the corresponding crystal, trap energies, and energy barriers.

The total solute content of cell $I$ at time step $k+1$

$$
H_{I}^{k+1}=H_{I}^{k}+\left(J_{I-\frac{1}{2}}^{k} A_{I-\frac{1}{2}}-J_{I+\frac{1}{2}}^{k} A_{I+\frac{1}{2}}\right) \Delta t
$$

is immediately obtained from Eqs. (16), (29), and (31). However, it is distributed in the different subregions of cell $I$

$$
H_{I}=H_{1}+H_{t}+H_{2},
$$

where $H_{i}(i=1,2)$ and $H_{t}$ are the amounts of solute to each side of the interface and trapped inside it, respectively. Forcing the solute in this cell to all be at the same chemical potential ignores the large discontinuities, which produces noticeable deviations from the correct solution. Instead, the amounts of solute in each subregion are obtained by integrating the concentration profiles between the evaluated points, assumed to be linear within the FVM scheme adopted. That is,

$$
H_{1}=\int_{x_{I-1}+\Delta x / 2}^{x_{I-1}+\phi_{1} \Delta x} \Psi(x)\left(\frac{c_{\Phi 1}-c_{I-1}}{\phi_{1} \Delta x}\left(x-x_{I-1}\right)+c_{I-1}\right) d x
$$

and

$$
H_{2}=\int_{x_{I-1}+\left(2-\phi_{2}\right) \Delta x}^{x_{I-1}+3 \Delta x / 2} \Psi(x)\left(\frac{c_{I+1}-c_{\Phi 2}}{\phi_{2} \Delta x}\left(x-x_{I+1}\right)+c_{I+1}\right) d x,
$$

where $\Psi(x)$ comes from the integration of $d V$ in the different coordinate systems; thus $\Psi=A, \Psi=2 \pi L x$ ( $L$ is the length of the cylindrical segment) and $\Psi=4 \pi x^{2}$ for cartesian, cylindrical and spherical coordinates, respectively. Substituting Eqs. (28) and (30) into (34) and (35), performing the integration and evaluating the limits leads to Eqs. of the form $H_{i}=m_{i} c_{i}+b_{i}$. Similarly, the amount of solute at the interface can be expressed as $H_{t}=m_{t} c_{t}$. Solving these equations together with (33) leads to the analytical solution

$$
c_{t}=\frac{H_{I}-b_{1}-b_{2}}{m_{t}+m_{1}+m_{2}},
$$

where $m_{t}=V_{t}$ is equal to the volume of the trap and the missing coefficients $m_{i}$ and $b_{i}$ are given in Appendix for the different coordinate systems.

The description of the current section can also be used to model an interface with a negative segregation, such as that shown in Fig. 3(b). While this is often described by a simpler energy barrier model like that of Sec. II, there could be a potential advantage in cases where the evolution of the concentration at the interface needs to be tracked.

\section{Multilayered interfaces}

All the previous effective coefficients of diffusion arise from a condition of equal flux throughout multiple regions of the atomic energy landscape. The results are consistent with the relation $D^{-1}=\sum_{i} \phi_{i} D_{i}^{-1}$ [50], also observed in multilayered materials [51-53], but also account for the difference in energy levels on both sides of the interface. By equating the fluxes within a domain of length $L$ consisting of $n$ individual atomic jumps between two energy levels, each of length $\phi_{b i} L$, the following relationship is reached:

$$
J_{\text {mult }}=-\hat{D}_{b, \text { mult }} \frac{\frac{c_{n}}{S_{n}}-\frac{c_{1}}{S_{1}} \exp \left(-\frac{E_{n}-E_{1}}{R T}\right)}{L},
$$

where the subindinces 1 and $n$ denote the properties at the two ends of such domain, and

$$
\hat{D}_{b, \mathrm{mult}}=\left(\sum_{i=1}^{n-1} \frac{\phi_{b i}}{\hat{D}_{b i}} \exp \left(-\frac{E_{n}-E_{i+1}}{R T}\right)\right)^{-1} .
$$

This can be thought of as the coefficient of diffusion for a configuration in series. Moreover, Eq. (38) reduces to the coefficients of diffusion in Eqs. (22), (27), (29), and (31) for the specific energy landscapes described before. Similarly, if the whole domain belongs to a single homogeneous material, Eqs. (37) and (38) become Fick's first law of diffusion.

A multilayered interface with arbitrary energy landscapes on both sides can be described with this approach and 


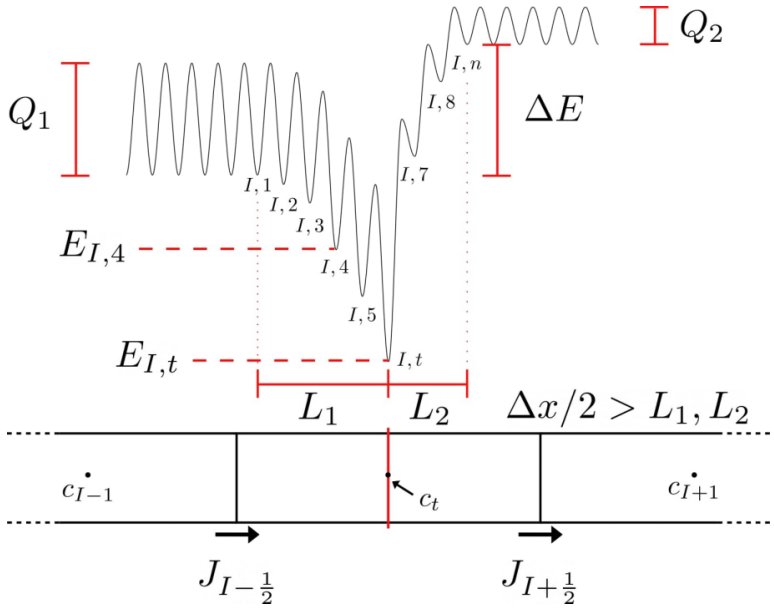

FIG. 4. Energy landscape (top) and cell discretization (bottom) of a multilayered interfacial trap. The individual atomic sites of the interface are labeled $(I, i)$ (for $i=1, \ldots, t, \ldots, n)$.

incorporated into the diffusion PDE. Consider, for instance, an interface like that in Fig. 4, where the center of cell $I$ at $x=x_{I}$ coincides with the atomic site with the lowest energy level at the interface. The concentration at that specific site is denoted $c_{t}$. The interface consists of $n$ different atomic sites, each at position $x_{I, i}$ with its corresponding volume $V_{I, i}$, site density $S_{I, i}$, diffusivity prefactor $D_{0 I, i}$ segregation energy $\Delta E_{1, i}=$ $E_{1}-E_{I, i}$ (where $\left.\Delta E_{1, i}=E_{1}-E_{I, i}\right)$, and concentration $c_{I, i}(i=1, \ldots, t, \ldots, n)$. The energy levels of the saddle points in between are denoted $E_{b, i}$ (where $\Delta E_{b 1, i}=E_{1}-E_{b, i}$ ). Points $I, 1$ and $I, n$ have the energy values of the neighboring crystals. The procedure of Sec. IIB3 can be followed by accounting for the thickness of the modeled interface $L=$ $L_{1}+L_{2}$, where $L_{1}<\Delta x / 2$ and $L_{2}<\Delta x / 2$. To determine the flux through the left boundary $J_{I-\frac{1}{2}}$, the new relevant length fractions become $\phi_{1}=1-\frac{L_{1}}{\Delta x}$ for crystal 1 and $\phi_{b 1}=\frac{L_{1}}{\Delta x}$ for the left segment of the interface. Solutions (28) and (29) are still valid, but require substituting the atomic jump coefficient of diffusion $\hat{D}_{b 1}$ for an equivalent one calculated via Eq. (38) between points $x_{I, 1}$ and $x_{I, t}$. The same can be done with the right segment of the interface, with the equivalent interface coefficient of diffusion between points $x_{I, t}$ and $x_{I, n}$.

A solute redistribution step is still required. The amount of solute trapped at the interface is

$$
H_{t}=\sum_{i=2}^{n-1} c_{I, i} V_{I, i} .
$$

Points 1 and $n$ are not included in the summation because they belong to the neighboring crystals rather than to the interface. For the sake of simplicity, a uniform chemical potential at the interface is assumed. This reasoning is sensible given the small thickness of the region where it is applied in comparison with the larger spatial domain modeled, plus being confined to the interfacial trap means that the inherent error associated does not scale with $\Delta x$. This assumption gives a set of equations of the form

$$
\frac{c_{I, i}}{S_{I, i}}=\frac{c_{t}}{S_{I, t}} \exp \left(-\frac{E_{I, i}-E_{I, t}}{R T}\right) .
$$

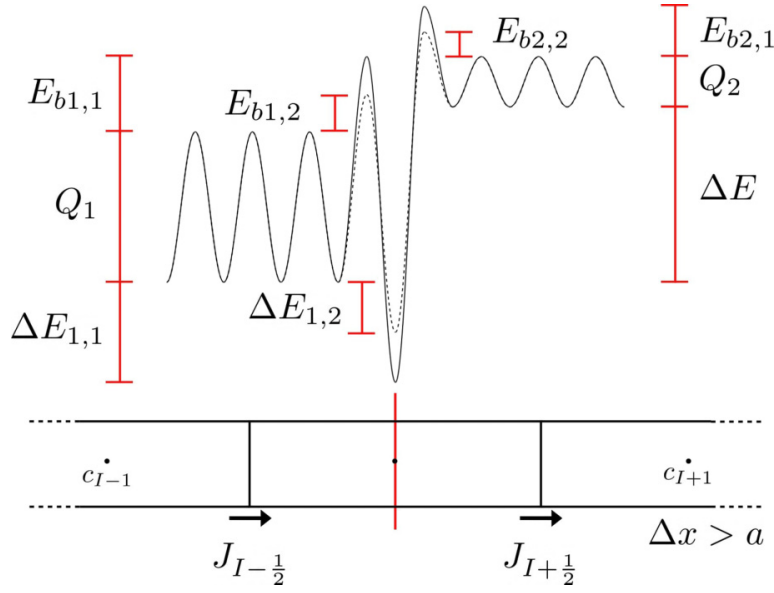

FIG. 5. Energy landscape (top) and cell discretization (bottom) of an heterogeneous interfacial trap. Two distinct interfacial site types are superimposed (dotted and solid lines), each with its respective segregation energies and energy barriers.

Solving Eqs. (39) and (40) leads to $H_{t}=m_{t} c_{t}$, with

$$
m_{t}=\frac{1}{S_{I, t}} \sum_{i=2}^{n-1} S_{I, i} V_{I, i} \exp \left(-\frac{E_{I, i}-E_{I, t}}{R T}\right) .
$$

The previously derived solution to update the trap concentration, Eq. (36), is still valid by considering the value of $m_{t}$ in Eq. (41).

\section{Heterogeneous interfaces}

Many bicrystal interfaces are not homogeneous, and they instead possess recognizable regions with different properties. For instance, think of semicoherent interfaces with both coherent regions and regularly spaced dislocations that make up for the lattice misfit of the crystals. Segregation will be favoured at the latter, while the former regions may behave in different ways.

A series of assumptions are suggested for this approach. Firstly, it is clear that heterogeneous interfaces would result in inhomogeneous lattice strains and corresponding segregation energies over a number of atomic planes. For simplicity, we consider the heterogeneities to be constrained to the monolayer interface, where we keep track of the concentrations at the different sites. Secondly, we assume that all sites at the interface are at thermodynamic equilibrium. This is promoted by energy barriers of similar heights and diffusion paths that interconnect the different interfacial sites, both of which are frequently present in real interfaces (e.g., Ref. [21]). The resulting energy landscape is schematically shown in Fig. 5, with a monolayer interface at the center of cell $I$ at $x=x_{I}$. The interface consists of $n$ distinct types of sites, each with its site density $S_{I, i}$, segregation energy $\Delta E_{1, i}$ (where $\Delta E_{1, i}=$ $E_{1}-E_{I, i}$, energy barriers on both crystals $E_{b 1, i}$ and $E_{b 2, i}$, and concentration $c_{I, i}(i=1, \ldots, n)$, all within the volume $V_{t}$ of the trap atomic layer.

Before considering diffusion across cells, the atomic jumps in the vicinity of the interface are required. Analogous to Eq. (21), there will now be $n$ fluxes from the left side (and also from the right side) of the interface into the individual 
sites, with the form

$$
J_{\Phi, i}=-\hat{D}_{b, i} \frac{\frac{c_{I, i}}{S_{I, i}}-\frac{c_{I^{-}}}{S_{1}} \exp \left(-\frac{E_{I, i}-E_{1}}{R T}\right)}{a},
$$

where $c_{I^{-}}$is the concentration at the atomic plane to the left of the interface and $D_{b, i}$ the individual atomic jump coefficient of diffusion calculated via Eq. (22). The driving forces of these equations are all the same, as an equal chemical potential is assumed at the interface. The total flux is the sum of all individual fluxes; thus, an equivalent coefficient of diffusion between the interface and the neighboring atomic layer is

$$
\hat{D}_{b, \text { het }}=\sum_{i=1}^{n}-\hat{D}_{b, i} \text {. }
$$

This can be thought of as the coefficient of diffusion for a configuration in parallel.

Lastly, the steps followed in Sec. IIB3 can be followed without major changes. Solutions (28) and (29), and equivalent ones for the segment to the right of the interface, are valid for the current approach, as long as the atomic jump coefficient of diffusion $\hat{D}_{b 1}$ is substituted for an equivalent one calculated via Eq. (43). Note the subindex ${ }_{t}$ in such equations must also be swapped for that of whichever site in the heterogeneous interface, in this case site ${ }_{n}$, to match nomenclatures. In a similar fashion to the redistribution step in Sec. IIB4, updating of the trap concentration after every step can be done with Eq. (36), but with a slope $m_{t}$ equal to

$$
m_{t}=\frac{V_{t}}{S_{I, n}} \sum_{i=1}^{n} S_{I, i} \exp \left(-\frac{E_{I, i}-E_{I, n}}{R T}\right) .
$$

\section{RESULTS AND DISCUSSION}

Multiple scenarios have been modeled in the current work, and the rationale behind their validation is as follows. We first examine in detail the atomic jump model, which represents the basic building block for all other cases. Then, for validation purposes, the results of simulations with the remaining cases are compared to those of the atomic jump model for a variety of energy landscapes obtained from reported literature of $\mathrm{H}$ segregation in metals (often studied to understand the deleterious phenomenon of $\mathrm{H}$ embrittlement [32]). The scalability of these approaches is investigated by tracking multiple fluxes and interfacial concentrations, where applicable, as $\Delta x$ increases in size. Relative errors are calculated with respect to the atomic jump model to determine the validity of the solutions. For generality, the models are also applied to some examples of $\mathrm{O}$ segregation in electronic devices. Lastly, parametric analyses and further discussions are given.

\section{A. Atomic jumps}

The derivation followed in Sec. IIB1 to determine the kinetics equation for the atomic jump model is consistent with classical approaches, although it is applied at the smallest length scale possible. While it considers fluxes at a continuum level, which subsequently enables its scalability, it recovers the solutions from atomistic derivations based on jump attempts [25,31] previously done only in single materials.
TABLE I. Parameters used by $\mathrm{Du}$ et al. [54] for the kMC simulations.

\section{Parameters}

$a=0.71 \AA$

$D_{0}=6.68 \times 10^{-8} \mathrm{~m}^{2} \mathrm{~s}^{-1}$

Energy barriers:

Bulk $\leftrightarrow$ bulk $0.088 \mathrm{eV}$

Bulk $\rightarrow$ interface $0.088 \mathrm{eV}$

Interface $\rightarrow$ bulk $0.6 \mathrm{eV}$

Interface $\leftrightarrow$ interface $0.25 \mathrm{eV}$

Yet, further validations are required for more complex energy landscapes.

Diffusion through model energy landscapes were studied by Du et al. [54] via a kinetic Monte Carlo (kMC) approach. These intended to mimic $\mathrm{H}$ diffusion through a combination of bulk bcc Fe and grain boundary configurations. They modeled different one-dimensional regions consisting of $l$ site layers, three of which at a lower energy level and with a different energy barrier between them representing the grain boundary. Employing periodic boundary conditions, they estimated effective diffusion coefficients $D_{\text {eff }}$ as time-weighted averages from the mean square displacements of the individual $\mathrm{H}$ atoms. The results of their simulations, using the parameters in Table I, are shown in Fig. 6(a). A constant site density was employed throughout the material, and results are independent of it.

Two approaches were followed to compare these results with the atomic jump model developed in the current work, both using the same parameters from Table I. Firstly, diffusion was simulated layer-by-layer with Eqs. (21) and (22) in large domains consisting of repeating units identical to the regions studied by Du. The initial concentration was set equal to zero everywhere except for a single point at the center situated in a bulk region, i.e., a Dirac delta function $c(x, 0)=\delta(x)$, and the simulated region was large enough to ensure the solute never reached the boundaries. The coefficient of diffusion was then calculated from the spread of the solute via the summation over all cells

$$
D_{\mathrm{eff} \delta}=\sum_{i} c_{i} \frac{x_{i}^{2}}{2 t} .
$$

Secondly, an estimation is made with the results from the multilayered interface model. Note that the diffusivity in Eq. (38) relates the flux with the concentration gradient in a given phase, whereas the one calculated by Du et al. is related to the average displacement of the $\mathrm{H}$ atoms. For both definitions to be equal, the former equation must be related to the gradient in the total concentration instead (a similar discussion was done by Jönsson et al. [55]). Thus, for a dilute solution in thermodynamic equilibrium in a material consisting of $n$ distinct regions each with volume fraction $\phi_{i}$,

$$
D_{\text {eff,mult }}=\hat{D}_{b, \text { mult }}\left(\sum_{i=1}^{n} \phi_{i} S_{i} \exp \left(-\frac{E_{i}-E_{1}}{R T}\right)\right)^{-1},
$$

where the subindex ${ }_{1}$ is here assigned to the bulk region. 

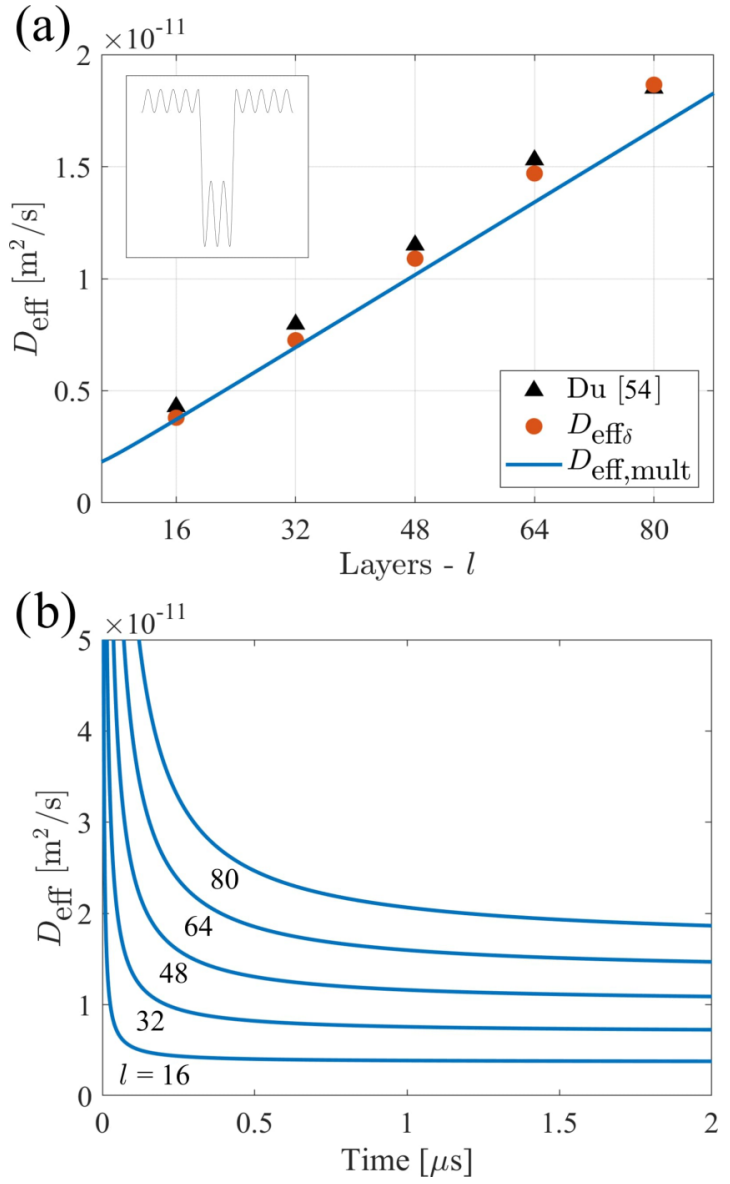

FIG. 6. (a) Effective coefficients of diffusion of the model landscapes simulated by Du et al. [54] and the estimates via a Dirac delta simulation and the multilayered approach. (b) Convergence of the Dirac delta simulation.

The results from both approaches, plotted in Fig. 6(a), should coincide. The estimated diffusivities from the Dirac delta approach are initially that from the bulk material but converge to lower values with time, as shown in Fig. 6(b). Despite of the long times of $2 \mu$ s these values did not reach saturation, particularly for the simulations with more layers pre repeating unit. Overall, there is a good agreement with Du's results. The small deviations may develop from the ideal and dilute approximations in the current model (interactions between $\mathrm{H}$ in nearby sites were included in the original work) or from not reaching saturation in the $\mathrm{kMC}$ simulation (just as in the Dirac delta case). Regardless, these results validate the use of the atomic jump flux equations (21) and (22) for heterogeneous energy landscapes.

The atomic jump model is key to modeling the remaining approaches in Sec. II. The best example is the case of a simple bicrystal interface, which results in either Eqs. (27) or (24), whether the atomic jump at the interface is included or not. The only difference accounts for the energy barrier over the length of a single site layer. When $\Delta x$ becomes larger, this effect becomes negligible as the coefficient of diffusion is weighed over many layers. However, ignoring this interfacial effects results in larger differences for small distances and large values of $\Delta E$. To the knowledge of the authors, (26) and
(27) are the first fully scalable equations to include the effects of the discontinuity at a solid-solid interface.

\section{B. Bicrystal interfaces \\ 1. $H$ in metals}

Diffusion of $\mathrm{H}$ through multiple bicrystal interfaces was simulated following the approaches introduced in Sec. II, with energy landscapes obtained from reported DFT studies. For each case, iterative algorithms from the discrete numerical solution (16) to the diffusion PDE (10) were simulated in Cartesian coordinate systems of size $L$ with the corresponding bicrystal interfaces at $x=L / 2$, Dirichlet boundary conditions $c(0, t)=1 \mathrm{~mol} \mathrm{~m}^{-3}$ and $c(L, t)=0 \mathrm{~mol} \mathrm{~m}^{-3}$, and an initial condition $c(x, 0)=0 \mathrm{~mol} \mathrm{~m}^{-3}$. Given the linearity of the approach employed, all the concentrations and fluxes calculated are directly proportional to that at the former boundary. A temperature of $573 \mathrm{~K}$ was arbitrarily selected for all cases. Simulations with a cell size equal to the site layer spacing, i.e., $\Delta x=a$, were performed employing the flux equations (21) at the bicrystal interface and (17) elsewhere. The length of the domain and the simulated time $t_{\text {end }}$ were chosen to ensure that the steady states were practically reached (with the exception of the heterogeneous interface case) while keeping the computation time reasonable. Additional simulations were then performed for larger values of $\Delta x$ using the corresponding flux equations for each case. The outputs $y_{m}$ of these models were assessed by calculating their relative errors compared to the outputs of the atomic jump model $y_{a j}$ as $\left(y_{m}-y_{a j}\right) / y_{a j}$. The relative errors from good performing models must remain low during the transient stage and converge to zero in the steady state. The time step sizes $\Delta t$ of each run were chosen to ensure that the convergence criterion $D \Delta t / \Delta x^{2}<1 / 2$ was fulfilled.

Different grain boundary characters have multiple effects on the $\mathrm{H}$ diffusion in pure fcc $\mathrm{Ni}$, as investigated by Di Stefano et al. [23]. H preferentially sits at octahedral intersticial sites in the bulk, and a $\Sigma 3\left(\begin{array}{lll}1 & 1 & 1\end{array}\right)[\overline{1} 10]$ symmetric tilt grain boundary behaves as an additional energy barrier to diffusion of $0.18 \mathrm{eV}$. This was modeled as a monolayer energy barrier with an energy landscape shown in Fig. 7(a), where the layer thickness corresponds to the distance between planes of octahedral interstitial sites. The parameters used are displayed in Table II. For the energy barrier model, Eq. (25) was employed to calculate the flux through the interface. Figure 7(a) shows concentration profiles developed after different times, with remarkable agreement between those from the atomic jump model and the one with the largest $\Delta x$. A small discontinuity produced by the grain boundary is observed at the center of the profile. The evolution of the fluxes through the bicrystal interface and the rightmost boundary are plotted in Figs. 7(b) and 7(c), both reaching the same steady state value as expected. The profiles from all the simulations were considerably close, so only that of the atomic jump is shown. The relative errors of all others with respect to that one are displayed in the inserts. These begin at -1 when the flux of the atomic jump model first appears and quickly stabilize at zero. The initially large errors occur only because the output of the atomic jump model $y_{a j}$ is very small. Note that the flux through the right boundary is calculated at the interface 

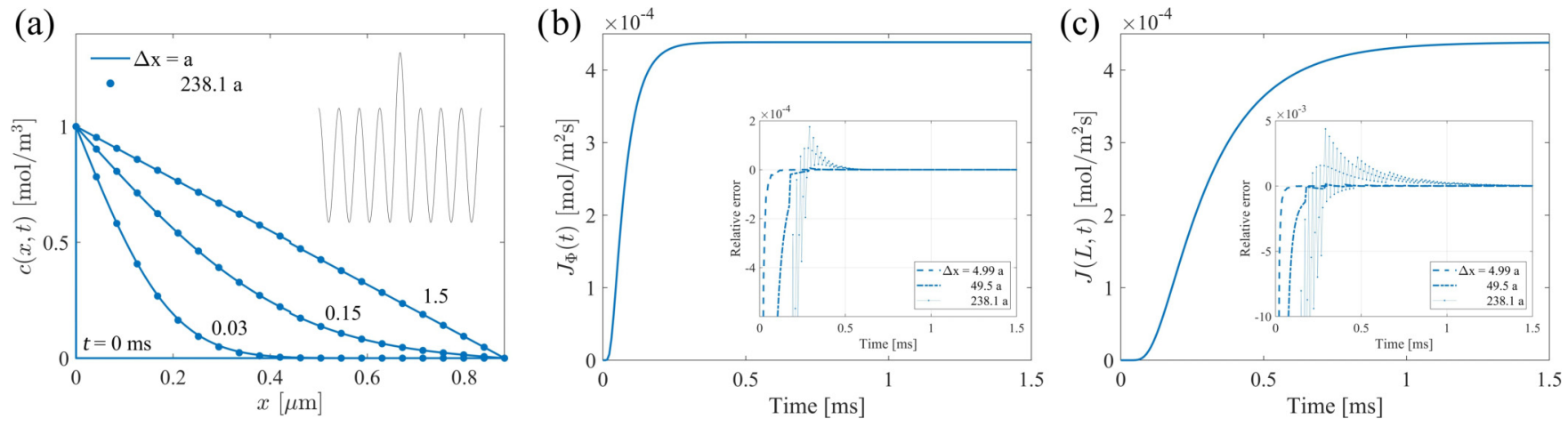

FIG. 7. Simulations of $\mathrm{H}$ diffusion through a $\Sigma 3\left(\begin{array}{lll}1 & 1 & 1\end{array}\right)\left[\begin{array}{lll}1 & 1 & 0\end{array}\right]$ grain boundary in fec Ni acting as an energy barrier to diffusion. (a) Concentration profiles and energy landscape, (b) evolution of the flux through the grain boundary and (c) through the right boundary. The inserts in (b) and (c) correspond to the relative errors of the simulations with larger $\Delta x$ with respect to that calculated via individual atomic jumps.

TABLE II. Parameters used in the $\mathrm{H}$ diffusion simulations.

\begin{tabular}{|c|c|c|c|}
\hline Case & Material parameters & & Simulation parameters \\
\hline $\begin{array}{l}\text { Energy barrier: } \\
\text { fcc } \mathrm{Ni} \\
\Sigma 3\left(\begin{array}{lll}1 & 1 & 1\end{array}\right)\left[\begin{array}{lll}\overline{1} & 1 & 0\end{array}\right]\end{array}$ & $\begin{array}{l}a=1.615 \AA \\
\Delta E=0 \\
D_{01}=D_{02}=7 \times 10^{-7} \mathrm{~m}^{2} \mathrm{~s}^{-1} \\
Q_{1}=Q_{2}=0.37 \mathrm{eV} \\
S_{1}=S_{2}=1.51 \times 10^{5} \mathrm{~mol} \mathrm{~m}^{-3} \\
E_{b 1}=E_{b 2}=0.18 \mathrm{eV}\end{array}$ & $\begin{array}{c}{[56]} \\
{[23]} \\
{[23]} \\
{[23]}\end{array}$ & $\begin{array}{l}L=5001 a \\
\Delta x=(a, 4.99 a, 49.5 a, 238.1 a) \\
t_{\text {end }}=1.5 \mathrm{~ms}\end{array}$ \\
\hline $\begin{array}{l}\text { Interfacial trap: } \\
\text { bcc Fe } \\
\Sigma 3\left(\begin{array}{lll}1 & 1 & 2\end{array}\right)\left[\begin{array}{lll}1 & \overline{1} & 0\end{array}\right]\end{array}$ & $\begin{array}{l}a=0.713 \AA \\
\Delta E=0 \\
D_{01}=D_{02}=1.38 \times 10^{-8} \mathrm{~m}^{2} \mathrm{~s}^{-1} \\
Q_{1}=Q_{2}=0.088 \mathrm{eV} \\
S_{1}=S_{2}=8.61 \times 10^{5} \mathrm{~mol} \mathrm{~m}^{-3} \\
\Delta E_{1 t}=0.43 \mathrm{eV} \\
D_{0 t}=D_{01} \\
S_{t}=2.39 \times 10^{5} \mathrm{~mol} \mathrm{~m}^{-3} \\
E_{b 1 t}=E_{b 2 t}=0 \mathrm{eV}\end{array}$ & $\begin{array}{c}{[57]} \\
{[22]} \\
{[22]} \\
{[22]} \\
\mathrm{b} \\
{[22]^{\mathrm{a}}} \\
\mathrm{b}\end{array}$ & $\begin{array}{l}L=5000 a \\
\Delta x=(a, 5 a, 50 a, 250 a) \\
t_{\text {end }}=0.1 \mathrm{~ms}\end{array}$ \\
\hline $\begin{array}{l}\text { Multilayered trap: } \\
\text { bcc Fe } \\
\Sigma 5\left(\begin{array}{lll}3 & 1 & 0\end{array}\right)\left[\begin{array}{lll}0 & 0 & 1\end{array}\right]\end{array}$ & $\begin{array}{l}a=0.713 \AA \\
\Delta E=0 \\
D_{01}=D_{02}=1.38 \times 10^{-8} \mathrm{~m}^{2} \mathrm{~s}^{-1} \\
Q_{1}=Q_{2}=0.088 \mathrm{eV} \\
S_{1}=S_{2}=8.61 \times 10^{5} \mathrm{~mol} \mathrm{~m}^{-3} \\
\Delta E_{1, i}=(0.13,0.5,0.13) \mathrm{eV} \\
D_{0 I, i}=\left(D_{01}, D_{01}, D_{01}\right) \\
S_{I, i}=(1.07,1.07,1.07) \times 10^{5} \mathrm{~mol} \mathrm{~m}^{-3} \\
E_{b 1, i}=(0.7,-0.7,-0.7,0.7) \mathrm{eV}\end{array}$ & $\begin{array}{l}{[22]} \\
{[57]} \\
{[22]} \\
{[22]} \\
{[22]} \\
b \\
{[22]^{\mathrm{a}}} \\
{[22]}\end{array}$ & $\begin{array}{l}L=5000 a \\
\Delta x=(a, 5 a, 50 a, 250 a) \\
t_{\text {end }}=0.2 \mathrm{~ms}\end{array}$ \\
\hline $\begin{array}{l}\text { Heterogeneous trap: } \\
\text { bcc Fe }+\mathrm{TiC} \\
\left(\begin{array}{lll}0 & 0 & 1\end{array}\right)_{\mathrm{Fe}} /\left(\begin{array}{lll}0 & 0 & 1\end{array}\right)_{\mathrm{TiC}} \\
{\left[\begin{array}{lll}1 & 0 & 0\end{array}\right]_{\mathrm{Fe}} / /\left[\begin{array}{lll}1 & 1 & 0\end{array}\right]_{\mathrm{TiC}}}\end{array}$ & $\begin{array}{l}a=0.713 \AA \\
\Delta E=0.68 \mathrm{eV} \\
D_{01}=1.38 \times 10^{-8} \mathrm{~m}^{2} \mathrm{~s}^{-1} \\
Q_{1}=0.088 \mathrm{eV} \\
S_{1}=8.61 \times 10^{5} \mathrm{~mol} \mathrm{~m}^{-3} \\
D_{02}=D_{01} \\
Q_{2}=0.47 \mathrm{eV} \\
S_{2}=6.5 \times 10^{5} \mathrm{~mol} \mathrm{~m}^{-3} \\
\Delta E_{1, i}=(0.32,0.49) \mathrm{eV} \\
D_{0 I, i}=\left(D_{01}, D_{01}, D_{01}\right) \\
S_{I, i}=(8.07,0.54) \times 10^{5} \mathrm{~mol} \mathrm{~m}^{-3} \\
E_{b 1, i}=(0,0) \mathrm{eV} \\
E_{b 2, i}=(0,0) \mathrm{eV}\end{array}$ & $\begin{array}{l}{[22]} \\
{[35]} \\
{[57]} \\
{[22]} \\
{[22]} \\
\mathrm{b} \\
{[35]} \\
{[35]} \\
{[35]} \\
\mathrm{b} \\
{[35]^{\mathrm{a}}} \\
\mathrm{b} \\
\mathrm{b}\end{array}$ & $\begin{array}{l}L=5000 a \\
\Delta x=(a, 5 a, 50 a, 250 a) \\
t_{\text {end }}=0.5 \mathrm{~ms}\end{array}$ \\
\hline
\end{tabular}

${ }^{\mathrm{a}}$ Calculated from data in that reference.

${ }^{\mathrm{b}}$ Assumed. 
(a)

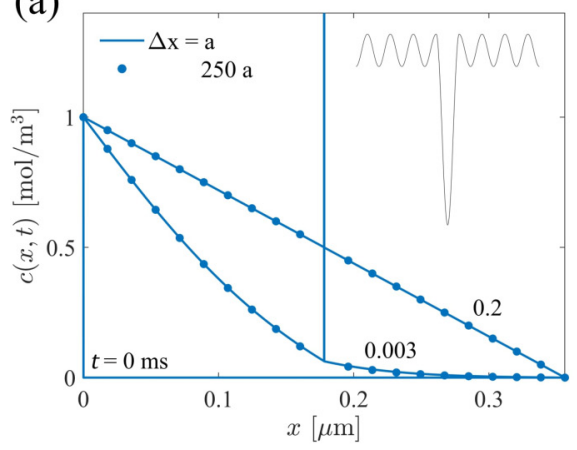

(b)

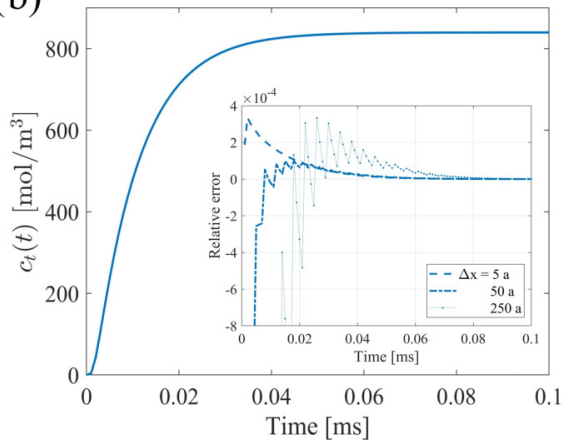

(c)

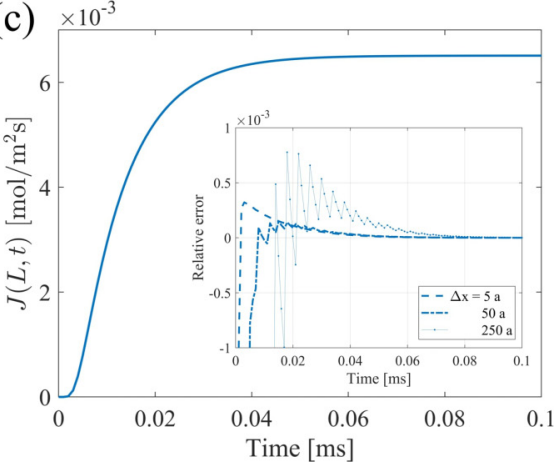

FIG. 8. Simulations of $H$ diffusion through a $\Sigma 3(112)[1 \overline{1} 0]$ grain boundary in bec Fe acting as an interfacial trap. (a) Concentration profiles and energy landscape, (b) evolution of the concentration at the interface site and (c) the flux through the right boundary. The inserts in (b) and (c) correspond to the relative errors of the simulations with larger $\Delta x$ with respect to that calculated via individual atomic jumps.

between the last two cells, the location of which slightly shifts to the left for larger cell sizes. Even with this consideration, the relative error remains considerably small.

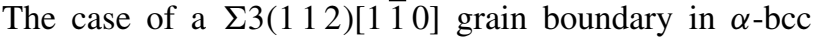
$\mathrm{Fe}$, investigated via DFT by Du et al. [22], is employed as an example of a monolayer interfacial trap. In the bulk, $\mathrm{H}$ sits preferentially at the tetrahedral sites, and there is a large segregation energy of $0.43 \mathrm{eV}$ at said interface, resulting in the energy landscape in Fig. 8(a). The volume used to calculate the trap site density $S_{t}$ is that of the interfacial area of the supercell employed in the original study times the spacing between planes of tetrahedral interstitial sites. Note that a site adjacent to the interface is reported to possess a segregation energy of $0.15 \mathrm{eV}$ [22], but this is ignored to employ the monolayer approach and because no information regarding the intermediate energy barriers is provided. Again, simulations for different $\Delta x$ values were done with the parameters given in Table II. The diffusivity prefactor in trap sites is assumed to be equal to that in the bulk and no additional energy barrier near the interface is considered. Other than that for $\Delta x=a$, the simulations employ the flux equations (29) and (31), and the solute redistribution step in Eq. (36). Concentration profiles at distinct times are shown in Fig. 8(a), again with remarkable agreement, where the large spikes extending in the vertical direction correspond to the trap concentration. The evolution of this parameter and the flux through the rightmost boundary are plotted in Figs. 8(b) and $8(\mathrm{c})$, respectively, together with the relative errors for the simulations with larger cell sizes. All these errors have the same behaviours as those described before, quickly stabilising around zero, which validates the scalability of the approach employed.

A $\Sigma 5(310)\left[\begin{array}{lll}0 & 0 & 1\end{array}\right]$ grain boundary in $\alpha$-bcc Fe is modeled here as a multilayered interface. The diffusion path to exit the site with the highest segregation energy of $0.5 \mathrm{eV}$ was also investigated by Du et al. [22]. The energy landscape is shown in Fig. 9(a), although this is still a simplification as other interfacial sites with a lower segregation energy are neglected, and the site density at the interface is also calculated from the corresponding supercell in the original study. The parameters employed for these simulations can be found in Table II. The flux equations (29) and (31) with an interfacial coefficient of diffusion calculated according to (38), together with the solute redistribution equations (36) and (41), are used to update the trap concentration. Some concentration profiles developed are shown in Fig. 9(a), which resemble those from the monolayer interfacial trap example. However, a larger concentration is reached in the trap site with the largest segregation energy, as shown in Fig. 9(b). The corresponding relative errors for the larger cell sizes converge in this case to $-2 \times 10^{-4}$ due to the assumption in Eq. (40) that imposes an equal chemical potential for all interfacial sites. The error is considerably small, but would be expected to increase for cases with larger energy barriers between the interfacial site layers. The flux through the rightmost boundary is shown in Fig. 9(c), with equally small relative errors for the simulations with larger cell sizes. This parameter saturates at a value almost identical to that in the monolayer trap case regardless of the larger segregation energy, indicating that the energy barrier to escape the trap does not affect the overall coefficient of diffusion. This is further explained in Sec. III C.

Lastly, the heterogeneous interface approach is validated with reported data on the interface between an $\alpha$-bcc Fe crystal and a TiC carbide, where $\mathrm{H}$ tends to partition to the former. A comprehensive DFT study by Di Stefano et al. [35] explored the energy landscape of the Baker-Nutting orientation relationship, $\left(\begin{array}{llll}0 & 0 & 1\end{array}\right)_{\mathrm{Fe}} /\left(\begin{array}{lll}0 & 0 & 1\end{array}\right)_{\mathrm{TiC}}$ and $\left[\begin{array}{lll}1 & 0 & 0\end{array}\right]_{\mathrm{Fe}} / \mid\left[\begin{array}{lll}1 & 1 & 0\end{array}\right]_{\mathrm{TiC}}$, previously observed experimentally [58,59]. The interface is composed of coherent regions, where the $\mathrm{Fe}$ and $\mathrm{C}$ atoms are aligned, and dislocations spaced every $4.6 \mathrm{~nm}$ [35], which corresponds to repeated units of one dislocation for every 15 atomic layers of $\alpha \mathrm{Fe}$. Only these two distinct regions are considered in the simulations for simplicity, even though a gradual transition between these would develop at the interface. In the coherent regions only the main trapping site with a segregation energy of $0.32 \mathrm{eV}$ is considered for the present analysis, as the original study did not explore the possibility of simultaneously occupying nearby sites. Additionally, a segregation energy of $0.49 \mathrm{eV}$ to sites within the dislocation core is considered. The parameters employed for the simulations are given in Table II and the resulting energy landscape superimposing the interfacial sites is shown in Fig. 10(a). Note that a constant site layer spacing $a$ (that of $\alpha \mathrm{Fe}$ ) was considered in the simulation because it only affects the coefficients of diffusion at the interfacial sites, which happen to be on the side of the iron crystal [35]. Besides, no value for the 

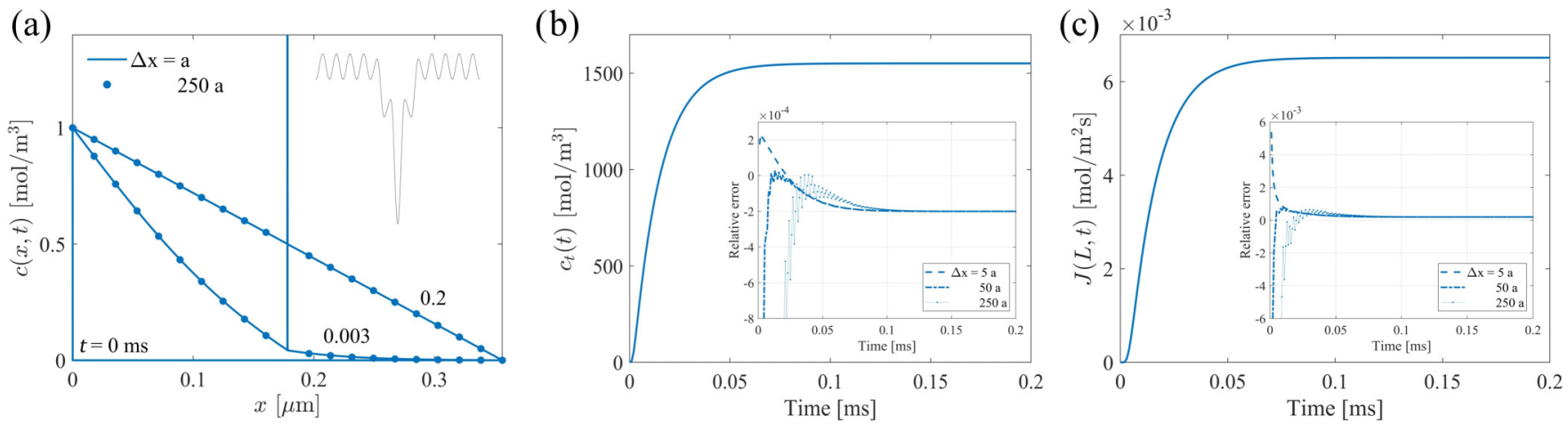

FIG. 9. Simulations of $\mathrm{H}$ diffusion through a $\Sigma 5(310)\left[\begin{array}{lll}0 & 1 & 1\end{array}\right]$ grain boundary in bcc Fe acting as a multilayered interfacial trap. (a) Concentration profiles and energy landscape, (b) evolution of the concentration at the interface site and (c) the flux through the right boundary. The inserts in (b) and (c) correspond to the relative errors of the simulations with larger $\Delta x$ with respect to that calculated via individual atomic jumps.

diffusivity prefactor for $\mathrm{TiC}$ can be found in the literature, so the same value as that in pure Fe is used considering that the order of magnitude of the coefficient of diffusion is predominantly dictated by the large activation energy in this phase. Two simulations were performed for the smallest cell size $\Delta x=a$, one where thermodynamic equilibrium was imposed between the two interfacial sites and another one in which atomic jumps were individually considered to each of the these, without any mass transfer between them. These approaches are hereby denoted "equilibrium" and "nonequilibrium", respectively. Note that this terminology does not refer to the thermodynamic equilibrium of the whole system, but just to the different assumptions for the connectivity of different sites at the interface. Simulations for larger cells were also performed, based on the interfacial flux, diffusivity and solute redistribution equations (29), (31), (36), (43), and (44).

There is a good agreement between all the simulations for the heterogeneous interface. The concentration profiles reached are shown in Figs. 10(a) and 10(b) at different magnifications. The large interfacial trapping, compared to bulk trapping in $\mathrm{TiC}$, is readily seen. Note that even after $0.5 \mu \mathrm{s}$ a steady state has not been reached, which would look as a straight line in the $\mathrm{TiC}$ side, due to the slow coefficient of diffusion in this phase. The evolution of the total concentration at the monolayer heterogeneous interfacial trap is plotted in Fig. 10(c), together with the relative errors with respect to the atomic jump equilibrium approach. Interestingly, the results of the nonequilibrium approach are very similar to the equilibrium ones, with near to $67.6 \%$ of the $\mathrm{H}$ at the interface located at the dislocation sites. This occurs as solute in the trap sites interacts with the layers on both sides of the interface, preventing large variations in the chemical potential of individual sites. Larger cell sizes promote errors that start at most at -0.097 and rapidly converge to zero, indicating a good approximation.

The heterogeneous interface model is particularly effective for cases where a few distinct trapping site types are responsible for the segregation behavior at the interface. However, this is not always the case. If the interface is considerably more heterogeneous and has no (or little) periodicity then this model would oversimplify the results. For instance, grain boundaries with second phases in them. Other potential problems for this model may be interfaces where sites with large segregation energies can only be accessed via paths with high energy barriers, in which case thermodynamic equilibrium with the neighboring atomic planes cannot be achieved. Comprehensive characteriza- (a)

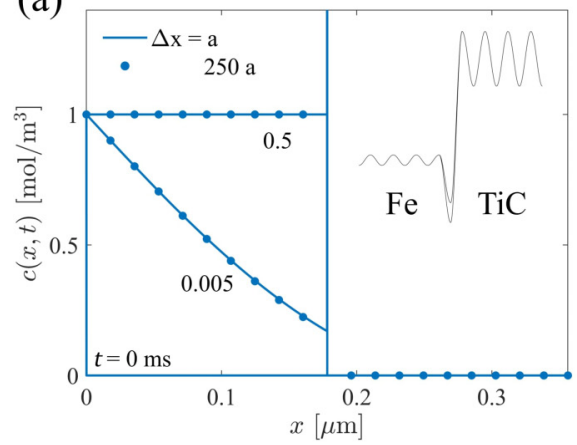

(b)

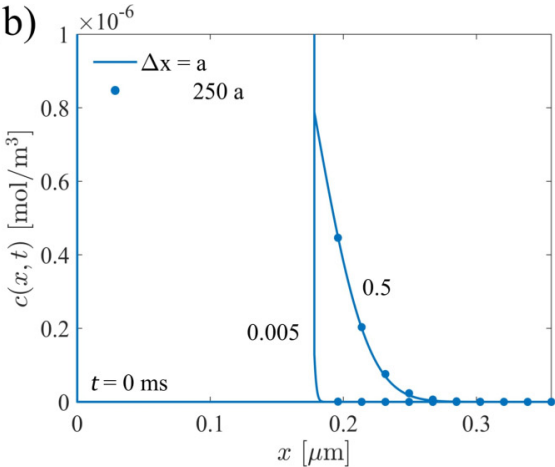

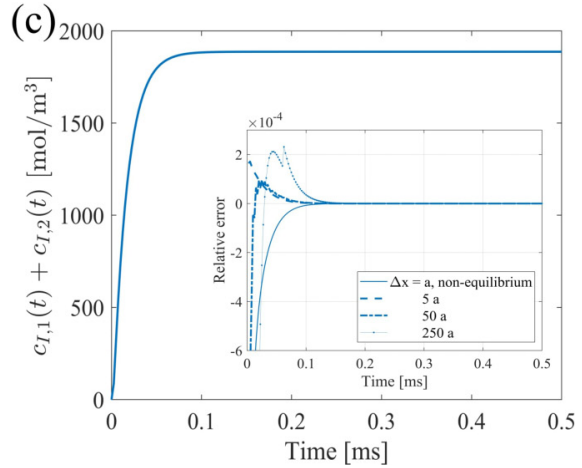

FIG. 10. Simulations of $\mathrm{H}$ diffusion through a $\left(\begin{array}{llll}0 & 0 & 1\end{array}\right)_{\mathrm{Fe}} /\left(\begin{array}{llll}0 & 0 & 1\end{array}\right)_{\mathrm{TiC}}$ and $\left[\begin{array}{lll}1 & 0 & 0\end{array}\right]_{\mathrm{Fe}} / \mid\left[\begin{array}{lll}1 & 1 & 0\end{array}\right]_{\mathrm{TiC}}$ bicrystal interface acting as a heterogeneous interfacial trap. (a) Energy landscape and concentration profiles in the Fe and (b) the TiC crystals, and (c) evolution of the total concentration at the interface. The insert in (c) corresponds to the relative error of the simulations with larger $\Delta x$, and the atomic jump nonequilibrium approach, with respect to the atomic jump equilibrium approach. 
TABLE III. Parameters used in the diffusion simulations of oxygen defects.

\begin{tabular}{|c|c|c|c|}
\hline Case & Material parameters & & Simulation parameters \\
\hline O interstitials: & $\begin{array}{l}a=2.55 \AA \\
\Delta E=-3.61 \mathrm{eV} \\
D_{01}=1.7 \times 10^{-9} \mathrm{~m}^{2} \mathrm{~s}^{-1} \\
Q_{1}=0.5 \mathrm{eV} \\
S_{1}=4.36 \times 10^{4} \mathrm{~mol} \mathrm{~m}^{-3} \\
D_{02}=2.1 \times 10^{-5} \mathrm{~m}^{2} \mathrm{~s}^{-1} \\
Q_{2}=2.26 \mathrm{eV} \\
S_{2}=1.63 \times 10^{5} \mathrm{~mol} \mathrm{~m}^{-3} \\
\Delta E_{1 t}=3.97 \mathrm{eV} \\
D_{0 t}=D_{02} \\
S_{t}=S_{2} \\
E_{b 1}=E_{b 2}=0\end{array}$ & $\begin{array}{c}{[60]} \\
{[60]} \\
{[63]^{\mathrm{b}}} \\
{[60]} \\
{[60]^{\mathrm{a}}} \\
{[64]} \\
{[60]} \\
{[60]^{\mathrm{a}}} \\
{[60]} \\
\mathrm{b} \\
{[60]^{\mathrm{a}}}\end{array}$ & $\begin{array}{l}L=21.46 \mathrm{~nm} \\
\Delta x=2.146 \mathrm{~nm} \\
T=293 \mathrm{~K} \\
t_{\text {end }}=2 \mathrm{~min} \\
\Delta t=0.49 \mathrm{~s}\end{array}$ \\
\hline O vacancies: & $\begin{array}{l}a=2.55 \AA \\
D_{01}=1.7 \times 10^{-9} \mathrm{~m}^{2} \mathrm{~s}^{-1} \\
Q_{1}=1.95 \mathrm{eV} \\
S_{1}=4.36 \times 10^{4} \mathrm{~mol} \mathrm{~m}^{-3} \\
\Delta E_{1 t}=1.08 \mathrm{eV} \\
D_{0 t}=D_{01} \\
S_{t}=S_{1} \\
E_{b 1}=-1.07 \mathrm{eV} \\
E_{b 2}=10^{20} \mathrm{eV}\end{array}$ & $\begin{array}{c}{[61]} \\
{[63]^{\mathrm{b}}} \\
{[61]} \\
{[61]^{\mathrm{a}}} \\
{[61]} \\
\mathrm{b} \\
{[61]^{\mathrm{a}}} \\
{[61]} \\
\mathrm{b}\end{array}$ & $\begin{array}{l}L=21.46 \mathrm{~nm} \\
\Delta x=2.146 \mathrm{~nm} \\
T=1023 \mathrm{~K} \\
t_{\text {end }}=10 \mathrm{~min} \\
\Delta t=4.93 \mathrm{~s}\end{array}$ \\
\hline
\end{tabular}

${ }^{\mathrm{a}}$ Calculated from data in that reference.

${ }^{\mathrm{b}}$ Assumed.

tions of the local energy landscapes are necessary to avoid this.

The above analyses exemplify diverse situations where the developed models can be applied. Besides, they demonstrate the scalability of the approach, which recovers the evolution of both segregation and long-range diffusion effects. For all cases, increases in the cell size of $\sim 250$ times, with their corresponding larger values of $\Delta t$, resulted in an average reduction of $99.997 \%$ of the computational time at the expense of the minor deviations shown. While only results of convergence for Cartesian coordinates were shown here, very similar results were obtained for cylindrical and spherical geometries.

\section{Point defects in electronic devices}

For the sake of generality, we also model the diffusion and trapping of point defects in electronic devices. Tang et al. applied DFT to describe the segregation energies and migration energy barriers of neutral oxygen interstitials [60] and vacancies [61] at a monoclinic $\mathrm{HfO}_{2}$ and Si interface for transistor applications. The heterostructure considered was built by aligning the [001] directions of both crystals and stretching the $\mathrm{HfO}_{2}$ crystal to match the lattice constant of Si. The defects considered have their origin during the deposition of $\mathrm{HfO}_{2}$, and their accumulation at the interface often results in the formation of unwanted phases.

No full description of the energy landscape from interface to bulk was developed in the original works, so the monolayer trap model is preferred here. The interfacial segregation energy of $\mathrm{O}$ interstitials has negligible dependence on the number of trapped atoms, and that for neutral $\mathrm{O}$ vacancies increases only slightly with it [62], which validates the use of the dilute solution approximation. No-flux Neu- mann boundary conditions $J(0, t)=J(L, t)=0 \mathrm{~mol} \mathrm{~m}^{-2} \mathrm{~s}^{-1}$ are implemented to determine the post-deposition redistribution of defects, with an initial reference concentration of $1 \mathrm{~mol} \mathrm{~m}{ }^{-3}$ only in $\mathrm{HfO}_{2}$. The simulations are performed in a domain with length of $21.46 \mathrm{~nm}$ with the interface in the middle, which corresponds to about $84 \mathrm{Hf}$ atomic layers. Such small domains are of interest for electronic device applications. All the parameters employed are given in Table III.

Oxygen interstitials in $\mathrm{HfO}_{2}$ bound to threefold coordinated lattice oxygen atoms have a migration energy barrier low enough that their redistribution is expected to occur even at room temperature. Unfortunately, the diffusivity prefactor has not been reported, so we use that for neutral vacancies considering that this does not affect the aim of these simulations to exemplify the applicability of the model. Figure 11(a) shows the energy landscape and the predicted kinetics of segregation at the first layer of Si below $\mathrm{HfO}_{2}$. After 2 minutes, most of the interstitial $\mathrm{O}$ atoms have segregated to the interface. Although further redistribution into the Si substrate would be favoured thermodynamically, the large diffusion energy barrier in this material prevents this from happening, promoting also the formation of a thin interfacial $\mathrm{SiO}_{x}(0<$ $x<2$ ) layer [60].

Neutral vacancies of fourfold coordinated $\mathrm{O}$ atoms have considerably larger migration energy barriers. Thus we model their redistribution over an annealing stage at $1023 \mathrm{~K}$. Note that doubly positively charged vacancies diffuse faster [65-67], but their interfacial segregation energy has not been reported. The diffusivity prefactor of the neutral defect is also assumed here to be that of the charged species. The insert in Fig. 11(b) shows the energy landscape modeled. O vacancies cannot exist in the $\mathrm{Si}$ side, so diffusion into this domain is prevented in the simulation by artificially setting a large 

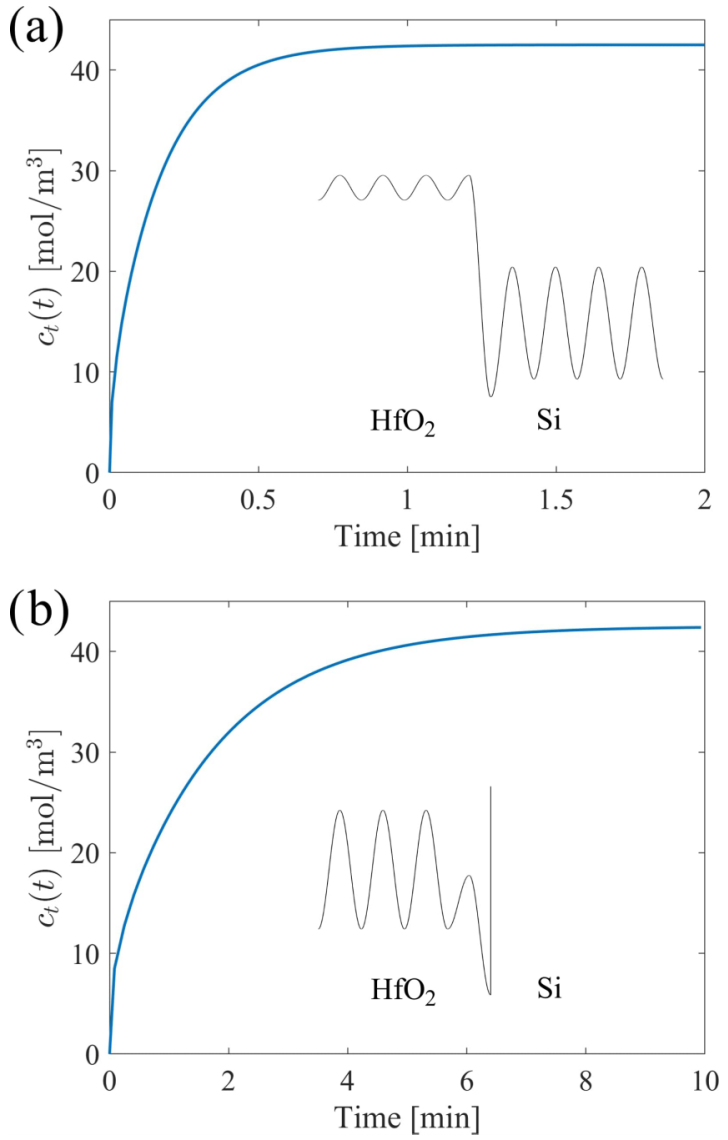

FIG. 11. Energy landscape and evolution of the concentration at the $\mathrm{HfO}_{2}$ and $\mathrm{Si}$ interface of (a) neutral O interstitials at $293 \mathrm{~K}$ and (b) neutral $\mathrm{O}$ vacancies at $1023 \mathrm{~K}$.

energy barrier after the last $\mathrm{HfO}_{2}$ layer $\left(E_{b 2}=10^{20} \mathrm{eV}\right)$. The smaller energy barrier near the interface makes an explicit atomic jump model $(\Delta x=a)$ prohibitively computationally expensive as $\Delta t$ must take very small values to converge just because of that individual cell. The monolayer trap can instead capture the diffusion kinetics, shown in Fig. 11(b), with a considerably larger time step. The segregation of vacancies to the interface, which may promote the formation of an interfacial layer of hafnium silicide [61], takes minutes in this scenario.

\section{Parametric analyses}

We now perform parametric analyses on key interfacial parameters to clarify their effects and further validate the approach. The simulations are done in a model energy landscape with the parameters from Table IV, with an interface situated at the center $x=L / 2$, where the diffusivity and site density are the same on both crystals but the solute partitions to the material in the left. The effects of varying energy barriers for an interface without segregation are shown in Fig. 12(a). For each simulation, the flux through the interface reaches saturation, followed by that through the rightmost boundary. As expected, larger energy barriers slow down the process and diminish the steady state flux reached, although not by the same amount. Clear effects can only be seen from $0.3 \mathrm{eV}$. This is better explained by enquiring the equivalent coefficient
TABLE IV. Parameters used for the simulations of the model bicrystals, unless otherwise specified.

\begin{tabular}{ll}
\hline \hline Material parameters & Simulation parameters \\
\hline$a=0.713 \AA$ & $L=10000 a$ \\
$\Delta E=0.5 \mathrm{eV}$ & $\Delta x=L / 30$ \\
$D_{01}=D_{02}=1.38 \times 10^{-8} \mathrm{~m}^{2} \mathrm{~s}^{-1}$ & $t_{\mathrm{end}}=0.4 \mathrm{~ms}$ \\
$Q_{1}=Q_{2}=0.088 \mathrm{eV}$ & $T=573 \mathrm{~K}$ \\
$S_{1}=S_{2}=8.61 \times 10^{5} \mathrm{~mol} \mathrm{~m}^{-3}$ & $c(0, t)=1 \mathrm{~mol} \mathrm{~m}^{-3}$ \\
& $c(L, t)=0$ \\
& $c(x, 0)=0$ \\
\hline \hline
\end{tabular}

of diffusion of the simulated region, obtained from Eq. (38) and plotted as a function of the energy barrier in Fig. 12(b). The effect of a small barrier is negligible, as its effect over a single atomic layer is weighted against numerous (99999 in this case) smaller jumps in the bulk of the crystals. With Eq. (37) one can recover the steady state fluxes reached in the simulations.

Consider now a monolayer interfacial trap instead, in the same model bicrystal. Increased concentrations develop for larger segregation energies, as expected, as shown in Fig. 13(a). Regardless, these saturate nearly around the same
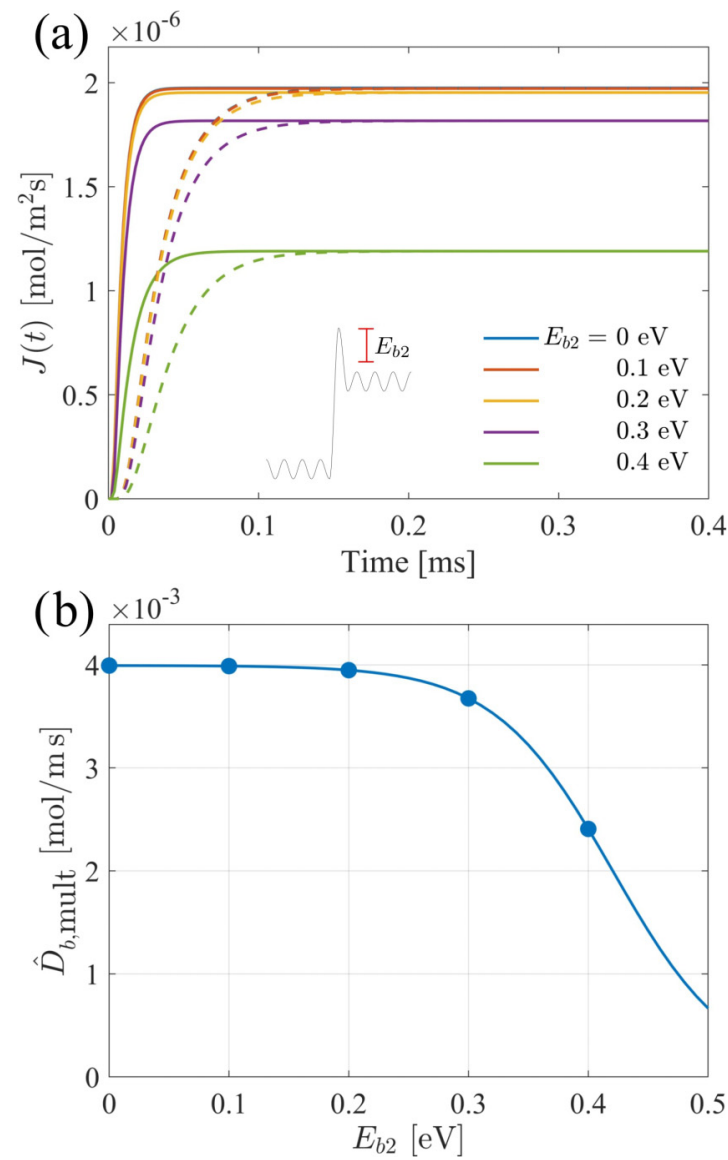

FIG. 12. (a) Effects of the interfacial energy barrier on the fluxes through the bicrystal interface (solid lines) and the rightmost boundary (dashed lines), and (b) on the equivalent coefficient of diffusion $\hat{D}_{b, \text { mult }}$ in the model bicrystal modeled. 

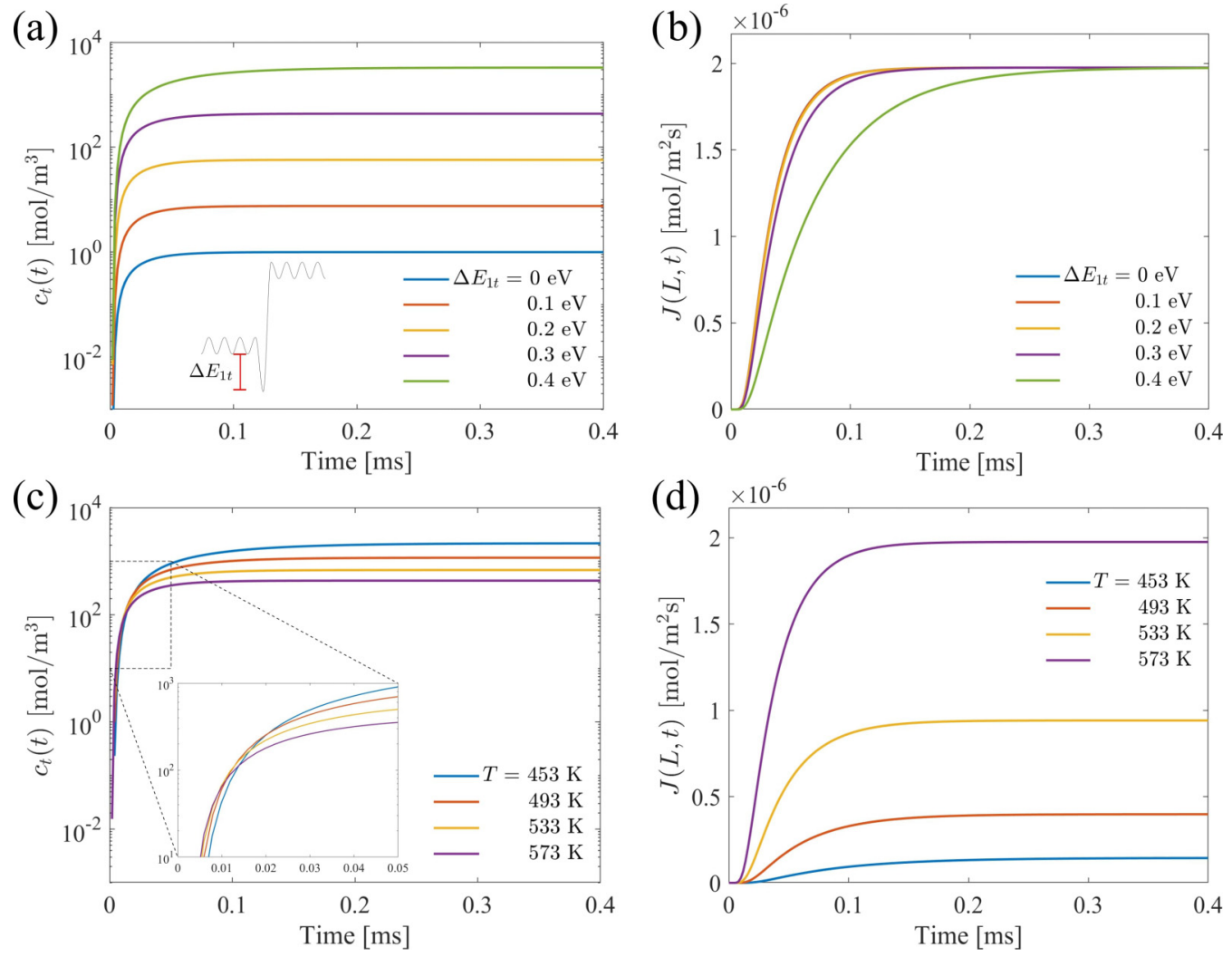

FIG. 13. Evolution of the [(a) and (c)] concentration at the interface and [(b) and (d)] fluxes through the rightmost boundary of the model bicrystal as a function of [(a) and (b)] interfacial segregation energy (at $573 \mathrm{~K}$ ) and [(c) and (d)] temperature (with $\Delta E_{1 t}=0.3 \mathrm{eV}$ ).

time due to the dependence of the driving force on such energy. Only the simulation with the largest segregation energy shows a noticeably slower saturation. This is better appreciated when examining the flux through the rightmost boundary in Fig. 13(b). It is important to note that the steady state flux is the same for all cases. While larger segregation energies imply bigger energy barriers to escape the interface, this effect is counteracted by the larger concentration in this region, i.e., more jump attempts with a lower success ratio results in the same original flux. The same can be deducted from Eq. (38).

The effects of temperature on the thermodynamic equilibrium and diffusion kinetics are shown in Figs. 13(c) and 13 (d) for a segregation energy of $0.3 \mathrm{eV}$. Higher segregations develop at lower temperatures, consistent with Eq. (9). The insert in Fig. 13(c) shows that trapping is slower at first, due to the slower diffusion kinetics, but it later overcomes that at higher temperatures due to the larger driving force. The solute fluxes through the rightmost boundary in Fig. 13(d) are also clearly larger at higher temperatures, as expected. Similar behaviours are anticipated for the multilayer and heterogeneous interface models.

While the examples used are still simplifications, they take us a step closer to the modeling of more realistic interfaces, the energy landscapes of which are likely combinations of the multilayered and heterogeneous models with numerous sites and energy barriers in between. The effects of different types of sites can be rationalized in the following way. Including in the analysis the sites at lower energy levels helps to recover the segregation kinetics at the interface. Alternatively, while those at higher energy levels have a little effect on the local concentration evolution, they act as paths of fast diffusion between the two crystals. Lastly, the energy barriers between all these sites at the interface will determine whether the concentrations in them evolve maintaining the same chemical potential.

\section{Final remarks}

The approach introduced in this work generalizes the treatment of solute diffusion at immobile solid-solid interfaces. This combines the accurate treatment of discontinuities in the energy landscape of reaction rate theory [25,26,31] and mobility-based equations [17,19] at a continuum level. In return, this allows for the calculation of diffusion and segregation rates for arbitrary bicrystal interfaces, boundary conditions and length scales. The effects intrinsic to the interface must be considered in systems where the segregation energy is not negligible, e.g., the local concentration at the $\mathrm{Fe}+\mathrm{TiC}$ interface becomes three orders of magnitude higher than that in the bulk.

An advantage of this approach is that it captures the solute kinetics accounting for first principle calculations of the energy landscape. The calculations performed for the different interfaces are examples of this. This bridge between scales opens the door to new applications of such atomistic data into realistic material conditions.

The analyses performed also have implications for the diffusion of $\mathrm{H}$ in structural alloys. Both types of grain boundaries, with energy barriers or interfacial trap sites, have a small effect on the $\mathrm{H}$ diffusivity upon reaching a steady state. 
However, the latter slow down the process during the transient regime as the segregation concentration evolves. These findings further support the point trap homogenization schemes, such as Oriani's [9] and McNabb-Foster's [68] models, for grain boundaries and second phases with no (or low) volume trapping. The difference in these models lies on whether the concentration at the trap remains at equilibrium with that in the bulk, which depends on the segregation rates to and from these defects. The approach developed in the current work could further investigate condition-specific rates and determine the validity of the thermodynamic equilibrium assumption in Oriani's model. There is also value in estimating the segregation rates to interfaces at smaller length scales. In some systems, the accumulation of solute at bicrystal interfaces impacts the formation of second phases (e.g., Ti hydrides at $\alpha / \beta$ interfaces in Ti alloys [69]). The implemented approach can be used to further study the nucleation of such phases.

Regarding potential future work, a number of assumptions currently in the model could be relaxed. For instance, the usage of the dilute and ideal solution approximation makes the PDE linear and allows for simple analytical equations to be obtained. This is an essential component of the classical Fick's laws of diffusion, although the assumption should be challenged when dealing with interfacial traps, as locally there is a lower number of available sites to accommodate the solute. There is potential to incorporate these effects by adapting the driving forces for diffusion, while performing similar analyses for the diffusivities at the interface. Moreover, diffusion along grain boundaries has relevance for different phenomena, and the corresponding energy barriers are often also extracted from DFT studies [22,23]. The concepts of diffusion in series and parallel in materials varying energy landscapes could potentially be applied in different directions to incorporate such effects. Full two- or three-dimensional models could also be attempted by performing further discretizations near the interfaces [70] and extending the solute redistribution steps for more complex geometries.

\section{CONCLUSIONS}

A fully scalable and thermodynamically consistent numerical solution was developed to model the diffusion and segregation kinetics through immobile solid-solid interfaces. This represents an important link between atomistic and macroscopic modeling of diffusion, and can be employed to investigate the effects of local variations in the energy landscape on physical phenomena across length scales. The approach works at a continuum level but includes discrete discontinuities in the diffusion parameters at the interface. The main findings are the following.

(1) An atomic jump kinetic equation is obtained from an equal flux condition at the smallest length scale valid. This is consistent with atomistic models based on individual jump attempts.

(2) Scaling up the above approach results in the first ever model to fully account for the discontinuity in the energy landscape at a bicrystal interface, therefore bridging the gaps in multiscale modeling of diffusion.

(3) The general models developed for arbitrary energy barriers and monolayer traps at bicrystal interfaces are able to track the evolution of the local solute segregation and long-range diffusion effects. These approaches are extended to deal with multilayered and heterogeneous interfaces, which are basic features of more realistic interfaces.

(4) Reported DFT diffusion data of $\mathrm{H}$ in structural metals and point defects in electronic devices are used as examples of the introduced models. The analyses provide more reliable rates of trapping and further validate the homogenization schemes that model grain boundaries as point traps.

\section{ACKNOWLEDGMENTS}

This work was part funded under the $£ 26 \mathrm{~m}$ Advanced Manufacturing and Materials (AMM) Programme as part of the Department for Business, Energy and Industrial Strategy's (BEIS) $£ 505 m$ Energy Innovation Programme in a bid led by Sheffield Forgemasters. The authors thank Jesus TalamantesSilva, Michael Blackmore, Michael Howson, and Jacob Pope at Sheffield Forgemasters for their support. We gratefully acknowledge the funding received from the EPSRC (Grant No. EP/T008687/1). E.I.G.-N. acknowledges funding from RAEng for his research fellowship.

\section{APPENDIX: EQUATIONS FOR THE SOLUTE REDISTRIBUTION STEP}

The equations for the parameters required in the solute redistribution step, Eq. (36) in Sec. IIB3, are given here for the three coordinate systems. To simplify the nomenclatures, the subindices 1 and 2 in this Appendix represent the cells to the left and right sides of the interface, respectively (e.g., $S_{1}=S_{I-1}$ and $\left.S_{2}=S_{I+1}\right)$.

(1) Cartesian coordinates (for $i=1,2$ ).

$$
\begin{gathered}
m_{i}=\frac{A \hat{D}_{b i} S_{i} \Delta x\left(-1+4 \phi_{i}^{2}\right)}{8 S_{t}\left(\hat{D}_{b i} \phi_{i}+D_{i} S_{i} \phi_{b i}\right)} \exp \left(-\frac{\Delta E_{i t}}{R T}\right), \\
b_{i}=\frac{A c_{i} \Delta x\left(-1+2 \phi_{i}\right)\left[\hat{D}_{b i}\left(-1+2 \phi_{i}\right)+4 D_{i} S_{i} \phi_{b i}\right]}{8\left(\hat{D}_{b i} \phi_{i}+D_{i} S_{i} \phi_{b i}\right)} .
\end{gathered}
$$

(2) Cylindrical coordinates.

$$
m_{1}=\frac{L \pi \hat{D}_{b 1} S_{1} \Delta x\left[3 x_{1}\left(-1+4 \phi_{1}^{2}\right)+\Delta x\left(-1+8 \phi_{1}^{3}\right)\right]}{12 S_{t}\left(\hat{D}_{b 1} \phi_{1}+D_{1} S_{1} \phi_{b 1}\right)} \exp \left(-\frac{\Delta E_{1 t}}{R T}\right),
$$




$$
\begin{gathered}
b_{1}=\frac{L \pi c_{1} \Delta x\left(-1+2 \phi_{1}\right)\left[\hat{D}_{b 1}\left(-1+2 \phi_{1}\right)\left(3 x_{1}+\Delta x+\phi_{1} \Delta x\right)+3 D_{1} S_{1} \phi_{b 1}\left(4 x_{1}+\Delta x+2 \phi_{1} \Delta x\right)\right]}{12\left(\hat{D}_{b 1} \phi_{1}+D_{1} S_{1} \phi_{b 1}\right)}, \\
m_{2}=\frac{L \pi \hat{D}_{b 2} S_{2} \Delta x\left[3 x_{1}\left(-1+4 \phi_{2}^{2}\right)+\Delta x\left(-5+24 \phi_{2}^{2}-8 \phi_{2}^{3}\right)\right]}{12 S_{t}\left(\hat{D}_{b 2} \phi_{2}+D_{2} S_{2} \phi_{b 2}\right)} \exp \left(-\frac{\Delta E_{2 t}}{R T}\right), \\
b_{2}=\frac{L \pi c_{2} \Delta x\left(-1+2 \phi_{2}\right)\left[\hat{D}_{b 2}\left(-1+2 \phi_{2}\right)\left(3 x_{1}+5 \Delta x-\phi_{2} \Delta x\right)+3 D_{2} S_{2} \phi_{b 2}\left(4 x_{1}+7 \Delta x-2 \phi_{2} \Delta x\right)\right]}{12\left(\hat{D}_{b 2} \phi_{2}+D_{2} S_{2} \phi_{b 2}\right)} .
\end{gathered}
$$

(3) Spherical coordinates.

$$
\begin{gathered}
m_{1}=\frac{\pi \hat{D}_{b 1} S_{1} \Delta x\left[24 x_{1}^{2}\left(-1+4 \phi_{1}^{2}\right)+16 x_{1} \Delta x\left(-1+8 \phi_{1}^{3}\right)+3 \Delta x^{2}\left(-1+16 \phi_{1}^{4}\right)\right]}{48 S_{t}\left(\hat{D}_{b 1} \phi_{1}+D_{1} S_{1} \phi_{b 1}\right)} \exp \left(-\frac{\Delta E_{1 t}}{R T}\right), \\
48\left(\hat{D}_{b 1} \phi_{1}+D_{1} S_{1} \phi_{b 1}\right) \\
b_{1}=\frac{\pi c_{1} \Delta x\left(-1+2 \phi_{1}\right)\left[\hat{D}_{b 1}\left(-1+2 \phi_{1}\right)\left(24 x_{1}^{2}+16 x_{1} \Delta x\left(1+\phi_{1}\right)+\Delta \phi_{b 1}\left(12 x_{1}^{2}+6 x_{1} \Delta x\left(1+2 \phi_{1}\right)+\Delta x^{2}\left(1+2 \phi_{1}+4 \phi_{1}^{2}\right)\right)\right]\right.}{\text { (A7) }}, \\
m_{2}=\frac{\pi \hat{D}_{b 2} S_{2} \Delta x\left[24 x_{1}^{2}\left(-1+4 \phi_{2}^{2}\right)+16 x_{1} \Delta x\left(-5+24 \phi_{2}^{2}-8 \phi_{2}^{3}\right)+\Delta x^{2}\left(-67+384 \phi_{2}^{2}-256 \phi_{2}^{3}+48 \phi_{2}^{4}\right)\right]}{48 S_{t}\left(\hat{D}_{b 2} \phi_{2}+D_{2} S_{2} \phi_{b 2}\right)} \exp \left(-\frac{\Delta E_{2 t}}{R T}\right),
\end{gathered}
$$

$b_{2}=\frac{\pi c_{2} \Delta x\left(-1+2 \phi_{2}\right)\left[\hat{D}_{b 2}\left(-1+2 \phi_{2}\right)\left(24 x_{1}^{2}-16 x_{1} \Delta x\left(-5+\phi_{2}\right)+\Delta x^{2}\left(67-28 \phi_{2}+4 \phi_{2}^{2}\right)\right)+8 D_{2} S_{2} \phi_{b 2}\left(12 x_{1}^{2}+6 x_{1} \Delta x\left(7-2 \phi_{2}\right)+\Delta x^{2}\left(37-22 \phi_{2}+4 \phi_{2}^{2}\right)\right)\right]}{48\left(\hat{D}_{b 2} \phi_{2}+D_{2} S_{2} \phi_{b 2}\right)}$.

[1] S. Fare, N. Lecis, E. Brescia, and M. Mazzola, Role of grain boundaries in diffusional phenomena during gas nitriding of pure iron, Procedia Eng. 10, 2943 (2011).

[2] M. Matsushita, Boronization and carburization of superplastic stainless steel and titanium-based alloys, Materials 4, 1309 (2011).

[3] C. Herzig, R. Willecke, and K. Vieregge, Self-diffusion and fast cobalt impurity diffusion in the bulk and in grain boundaries of hexagonal titanium, Philos. Mag. A 63, 949 (1991).

[4] E. Budke, C. Herzig, and H. Wever, Volume and grain boundary diffusion of 14C in $\alpha$-Fe, Phys. Status Solidi (a) 127, 87 (1991).

[5] Y. Mishin, C. Herzig, J. Bernardini, and W. Gust, Grain boundary diffusion: fundamentals to recent developments, Int. Mater. Rev. 42, 155 (1997).

[6] C. Yu, S. Ganapathy, E. R. van Eck, L. van Eijck, N. de Klerk, E. M. Kelder, and M. Wagemaker, Investigation of Li-ion transport in Li7P3S11 and solid-state lithium batteries, J. Energy Chem. 38, 1 (2019).

[7] S. Lou, Q. Liu, F. Zhang, Q. Liu, Z. Yu, T. Mu, Y. Zhao, J. Borovilas, Y. Chen, M. Ge, X. Xiao, W.-K. Lee, G. Yin, Y. Yang, X. Sun, and J. Wang, Insights into interfacial effect and local lithium-ion transport in polycrystalline cathodes of solid-state batteries, Nat. Commun. 11, 5700 (2020).

[8] K. Hadidi, T. Norby, O. Løvvik, and A. Gunnæs, Hydrogen energetics and charge transfer in the $\mathrm{Ni} / \mathrm{LaNbO} 4$ interface from DFT calculations, Int. J. Hydrogen Energy 37, 8033 (2012).

[9] R. Oriani and P. Josephic, Equilibrium aspects of hydrogeninduced cracking of steels, Acta Metall. 22, 1065 (1974).

[10] J. Li, C. Lu, L. Pei, C. Zhang, and R. Wang, Atomistic investigation of hydrogen induced decohesion of Ni grain boundaries, Mech. Mater. 150, 103586 (2020).
[11] M. Koyama, C. C. Tasan, E. Akiyama, K. Tsuzaki, and D. Raabe, Hydrogen-assisted decohesion and localized plasticity in dual-phase steel, Acta Mater. 70, 174 (2014).

[12] D. Barba, E. Alabort, S. Pedrazzini, D. Collins, A. Wilkinson, P. Bagot, M. Moody, C. Atkinson, A. Jérusalem, and R. Reed, On the microtwinning mechanism in a single crystal superalloy, Acta Mater. 135, 314 (2017).

[13] F. León-Cázares, F. Monni, and C. Rae, Stress orientation dependence for the propagation of stacking faults and superlattice stacking faults in nickel-based superalloys, Acta Mater. 199, 209 (2020)

[14] L. Darken, Diffusion of carbon in austenite with a discontinuity in composition, Transaction of the American Institute of Mining and Metallurgical Engineers 180, 430 (1949).

[15] J. W. Cahn, On spinodal decomposition, Acta Metall. 9, 795 (1961).

[16] M. Hillert, A solid-solution model for inhomogeneous systems, Acta Metall. 9, 525 (1961).

[17] L. Darken, Diffusion, mobility and their interrelation through free energy in binary metallic systems, Transactions of the American Institute of Mining and Metallurgical Engineers 175, 184 (1948).

[18] J. Ågren, Numerical treatment of diffusional reactions in multicomponent alloys, J. Phys. Chem. Solids 43, 385 (1982).

[19] J. Andersson and J. Ågren, Models for numerical treatment of multicomponent diffusion in simple phases, J. Appl. Phys. 72, 1350 (1992).

[20] D. McLean, Grain Boundaries in Metals (Oxford University Press, Oxford, 1957), p. 347.

[21] A. Pedersen and H. Jónsson, Simulations of hydrogen diffusion at grain boundaries in aluminum, Acta Mater. 57, 4036 (2009). 
[22] Y. A. Du, L. Ismer, J. Rogal, T. Hickel, J. Neugebauer, and R. Drautz, First-principles study on the interaction of $\mathrm{H}$ interstitials with grain boundaries in $\alpha$ - and $\gamma$-Fe, Phys. Rev. B 84, 144121 (2011).

[23] D. Di Stefano, M. Mrovec, and C. Elsässer, First-principles investigation of hydrogen trapping and diffusion at grain boundaries in nickel, Acta Mater. 98, 306 (2015).

[24] C. Lea and M. P. Seah, Kinetics of surface segregation, Philos. Mag. 35, 213 (1977).

[25] S. Hofmann and J. Erlewein, A model of the kinetics and equilibria of surface segregation in the monolayer regime, Surf. Sci. 77, 591 (1978).

[26] J. du Plessis and G. van Wyk, A model for surface segregation in multicomponent alloys-part III: The kinetics of surface segregation in a binary alloy, J. Phys. Chem. Solids 50, 237 (1989).

[27] J. du Plessis and G. Van Wyk, A model for surface segregation in multicomponent alloys-part IV: The kinetics of desegregation in a binary alloy, J. Phys. Chem. Solids 50, 247 (1989).

[28] M. Eisl and H. Störi, Surface segregation kinetics in binary substitutional systems: a comparison between MDE and KTBIM, Surf. Sci. 328, 135 (1995).

[29] J. du Plessis and G. van Wyk, A model for surface segregation in multicomponent alloys-part V: The kinetics of surface segregation in multicomponent alloys, J. Phys. Chem. Solids 50, 251 (1989).

[30] G. Martin, Atomic mobility in Cahn's diffusion model, Phys. Rev. B 41, 2279 (1990).

[31] D. N. Ilin, A. A. Kutsenko, D. Tanguy, and J.-M. Olive, Effect of grain boundary trapping kinetics on diffusion in polycrystalline materials: hydrogen transport in Ni, Modell. Simul. Mater. Sci. Eng. 24, 035008 (2016).

[32] H. K. D. H. Bhadeshia, Prevention of hydrogen embrittlement in steels, ISIJ Int. 56, 24 (2016).

[33] D. Gaude-Fugarolas, A comprehensive study of hydrogen redistribution and embrittlement prevention in ferrous alloys, in Frontiers in Materials Processing, Applications, Research and Technology (Springer Singapore, Singapore, 2018), pp. 203-219.

[34] S. Frappart, X. Feaugas, J. Creus, F. Thebault, L. Delattre, and $\mathrm{H}$. Marchebois, Study of the hydrogen diffusion and segregation into Fe-C-Mo martensitic HSLA steel using electrochemical permeation test, J. Phys. Chem. Solids 71, 1467 (2010).

[35] D. Di Stefano, R. Nazarov, T. Hickel, J. Neugebauer, M. Mrovec, and C. Elsässer, First-principles investigation of hydrogen interaction with TiC precipitates in $\alpha$-Fe, Phys. Rev. B 93, 184108 (2016).

[36] J. du Plessis and G. van Wyk, A model for surface segregation in multicomponent alloys-part I: Equilibrium segregation, J. Phys. Chem. Solids 49, 1441 (1988).

[37] J. du Plessis and G. van Wyk, A model for surface segregation in multicomponent alloys-part II: Comment on other segregation analyses, J. Phys. Chem. Solids 49, 1451 (1988).

[38] P. Lejček, in Climate Change 2013 - The Physical Science Basis, edited by Intergovernmental Panel on Climate Change, Springer Series in Materials Science, Vol. 136 (Springer Berlin Heidelberg, Berlin, Heidelberg, 2010), pp. 1-30.

[39] P. Lejček, L. Zheng, S. Hofmann, and M. Šob, Applied thermodynamics: Grain boundary segregation, Entropy 16, 1462 (2014).
[40] P. Wynblatt and D. Chatain, Anisotropy of segregation at grain boundaries and surfaces, Metall. Mater. Trans. A 37, 2595 (2006).

[41] P. Lejček and S. Hofmann, Modeling grain boundary segregation by prediction of all the necessary parameters, Acta Mater. 170, 253 (2019).

[42] R. Kirchheim, Lattice discontinuities affecting the generation and annihilation of diffusible hydrogen and vice versa, Philos. Trans. R. Soc. A 375, 20160403 (2017).

[43] J. Crank, The Mathematics of Diffusion (Oxford University Press, Oxford, 1956).

[44] P. Lejček, M. Šob, and V. Paidar, Interfacial segregation and grain boundary embrittlement: An overview and critical assessment of experimental data and calculated results, Prog. Mater. Sci. 87, 83 (2017).

[45] J. Peiró and S. Sherwin, Finite difference, finite element and finite volume methods for partial differential equations, in Handbook of Materials Modeling (Springer Netherlands, Dordrecht, 2005), pp. 2415-2446.

[46] R. Eymard, T. Gallouët, and R. Herbin, Finite volume methods, in Handbook of Numerical Analysis (Elsevier, 2000), pp. 713-1018.

[47] E. Elmukashfi, E. Tarleton, and A. C. F. Cocks, A modelling framework for coupled hydrogen diffusion and mechanical behaviour of engineering components, Comput. Mech. 66, 189 (2020).

[48] F. Boyer and F. Hubert, Finite volume method for 2D linear and nonlinear elliptic problems with discontinuities, SIAM J. Numer. Anal. 46, 3032 (2008).

[49] O. Farago, Algorithms for Brownian dynamics across discontinuities, J. Comput. Phys. 423, 109802 (2020).

[50] R. Hickson, S. Barry, G. Mercer, and H. Sidhu, Finite difference schemes for multilayer diffusion, Math. Comput. Model. 54, 210 (2011).

[51] D. Hasselman and L. F. Johnson, Effective thermal conductivity of composites with interfacial thermal barrier resistance, J. Compos. Mater. 21, 508 (1987).

[52] C.-W. Nan, R. Birringer, D. R. Clarke, and H. Gleiter, Effective thermal conductivity of particulate composites with interfacial thermal resistance, J. Appl. Phys. 81, 6692 (1997).

[53] J. Kalnin, E. Kotomin, and J. Maier, Calculations of the effective diffusion coefficient for inhomogeneous media, J. Phys. Chem. Solids 63, 449 (2002).

[54] Y. A. Du, J. Rogal, and R. Drautz, Diffusion of hydrogen within idealized grains of bcc Fe: A kinetic Monte Carlo study, Phys. Rev. B 86, 174110 (2012).

[55] B. Jönsson, H. Wennerström, P. G. Nilsson, and P. Linse, Selfdiffusion of small molecules in colloidal systems, Colloid \& Polymer Science 264, 77 (1986).

[56] M. Louthan, J. Donovan, and G. Caskey, Hydrogen diffusion and trapping in nickel, Acta Metall. 23, 745 (1975).

[57] Y. He, Y. Li, C. Chen, and H. Yu, Diffusion coefficient of hydrogen interstitial atom in $\alpha$-Fe, $\gamma$-Fe and $\epsilon$-Fe crystals by first-principle calculations, Int. J. Hydrogen Energy 42, 27438 (2017).

[58] F. G. Wei and K. Tsuzaki, Hydrogen absorption of incoherent TiC particles in iron from environment at high temperatures, Metall. Mater. Trans. A 35, 3155 (2004). 
[59] F. G. Wei and K. Tsuzaki, Quantitative analysis on hydrogen trapping of TiC particles in steel, Metall. Mater. Trans. A 37, 331 (2006).

[60] C. Tang and R. Ramprasad, Ab initio study of oxygen interstitial diffusion near Si:HfO2 interfaces, Phys. Rev. B 75, 241302(R) (2007).

[61] C. Tang, B. Tuttle, and R. Ramprasad, Diffusion of O vacancies near Si:HfO2 interfaces: An ab initio investigation, Phys. Rev. B 76, 073306 (2007).

[62] C. Tang and R. Ramprasad, Oxygen defect accumulation at Si:HfO2 interfaces, Appl. Phys. Lett. 92, 182908 (2008).

[63] M. Schie, M. P. Müller, M. Salinga, R. Waser, and R. A. De Souza, Ion migration in crystalline and amorphous HfO X, J. Chem. Phys. 146, 094508 (2017).

[64] C. Haas, The diffusion of oxygen in silicon and germanium, J. Phys. Chem. Solids 15, 108 (1960).

[65] N. Capron, P. Broqvist, and A. Pasquarello, Migration of oxygen vacancy in $\mathrm{HfO} 2$ and across the $\mathrm{HfO} 2 / \mathrm{SiO} 2$ interface:
A first-principles investigation, Appl. Phys. Lett. 91, 192905 (2007).

[66] S. Zafar, H. Jagannathan, L. F. Edge, and D. Gupta, Measurement of oxygen diffusion in nanometer scale $\mathrm{HfO} 2$ gate dielectric films, Appl. Phys. Lett. 98, 152903 (2011).

[67] S. Clima, Y. Y. Chen, R. Degraeve, M. Mees, K. Sankaran, B Govoreanu, M. Jurczak, S. De Gendt, and G. Pourtois, Firstprinciples simulation of oxygen diffusion in HfO $\mathrm{x}$ : Role in the resistive switching mechanism, Appl. Phys. Lett. 100, 133102 (2012).

[68] A. McNabb and P. K. Foster, A new analysis of diffusion of hydrogen in iron and ferritic steels, Trans. Metall. Soc. AIME 227, 618 (1963).

[69] B. Pound, Hydrogen trapping in aged $\beta$-titanium alloys, Acta Mater. 45, 2059 (1997).

[70] C.-N. Tzou and S. N. Stechmann, Simple second-order finite differences for elliptic PDEs with discontinuous coefficients and interfaces, Commun. Appl. Math. Comput. Sci. 14, 121 (2019). 